\title{
Chaos near a Resonant Inclination-Flip
}

\author{
Marcus Fontaine, William Kalies*, and Vincent Naudot
}

June 15, 2016

\begin{abstract}
Horseshoes play a central role in dynamical systems and are observed in many chaotic systems. However most points in a neighborhood of the horseshoe escape after finitely many iterations. In this work we construct a new model by re-injecting the points that escape the horseshoe. We show that this model can be realized within an attractor of a flow arising from a three-dimensional vector field, after perturbation of an inclination-flip homoclinic orbit with a resonance. The dynamics of this model, without considering the re-injection, often contains a cuspidal horseshoe with positive entropy, and we show that for a computational example the dynamics with re-injection can have more complexity than the cuspidal horseshoe alone.
\end{abstract}

\section{Introduction}

Smale's horseshoe map was introduced as an example of a chaotic and hyperbolic dynamical system which is topologically transitive and contains a countable set of periodic orbits [37]. The maximal invariant set of this map, the horseshoe, is the topological product of two Cantor sets, and the dynamics on this set is chaotic. The complement of the horseshoe is dense in a neighborhood so that nearby points escape after finitely many iterates. This model is observed when the stable manifold and the unstable manifold of a hyperbolic fixed point of a two-dimensional diffeomorphism intersect transversally [30]. This type of dynamics is also observed in the Poincaré return map of some three-dimensional vector fields, see the discussion below.

In this article we propose a model that contains similar features, but also dynamics with more complexity. This model is essentially obtained by re-injecting the points that escape a neighborhood of the horseshoe. We show that this model can be realized by considering the unfolding of a three-dimensional vector field that possesses two homoclinic orbits to the same resonant, hyperbolic equilibrium point, one of which is degenerate and the other is nondegenerate.

Homoclinic orbits can play an important role in the dynamics of flows; the corresponding dynamics is structurally unstable and therefore may lead to dramatic changes in the dynamics. The complexity of the dynamics obtained after perturbation is often related to the degeneracy of the unperturbed system, i.e. the number or parameters that are necessary for a typical unfolding.

Degenerate dynamics involving homoclinic orbits have received a lot of interest over the last few decades. Shil'nikov shows the presence of chaos near a homoclinic orbit when the linearization matrix at the saddle point has complex eigenvalues under certain conditions, see [39]. When the linearization has three real eigenvalues, Deng [6] shows that the unfolding of a degenerate, critically twisted, homoclinic orbit can lead to a suspended horseshoe. Deng describes a bifurcation scenario where the horseshoe is destroyed (or created) after infinitely many homoclinic bifurcations. Following this scenario, chaotic dynamics are observed, and the corresponding invariant set is called a cuspidal horseshoe, described in Figure 1. It is shown that a suspended cuspidal horseshoe can be realized in the unfolding of a degenerate homoclinic orbit in $\mathbb{R}^{3}$. In [6], critically twisted can mean two possible configurations - the orbit-flip and the inclination-flip, see below for more details and definitions.

In [17], the authors show that the scenario presented by Deng is possible in the case of an inclination-flip homoclinic orbit, as long as the unperturbed system satisfies some open condition. The authors study the Poincaré return map $\Psi$ on a cross section. For typical values of the parameters, the corresponding dynamics generalize that of Smale's horseshoe. Restricted to the maximal invariant set $\Lambda$, the Poincaré return map

\footnotetext{
*The second author was partially supported by NSF grant NFS-DMS-0914995.
} 

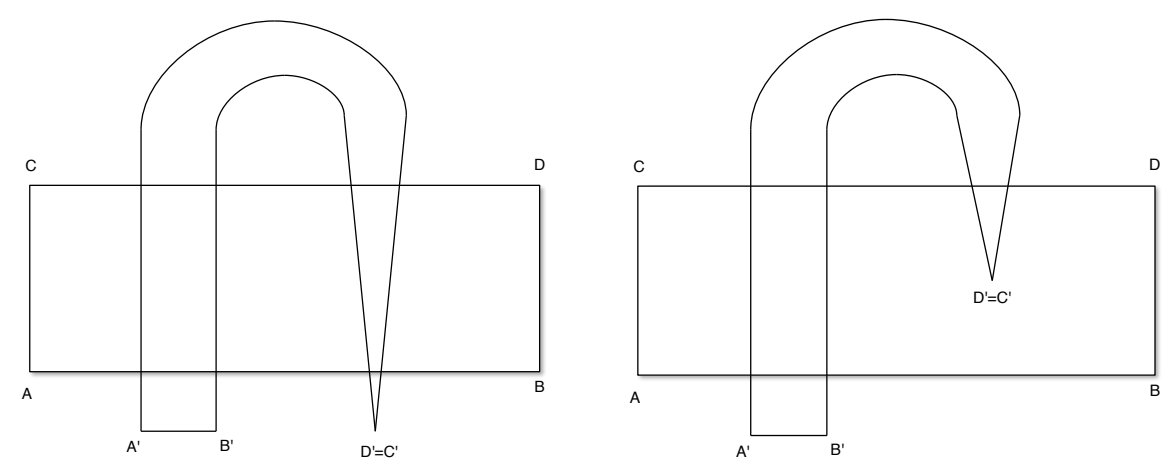

Figure 1: Two possible configurations of a cuspidal horseshoe. On the left, the dynamics is a full shift on two symbols. On the right, there is only a partial shift, which may or may not be chaotic.

is conjugate to a partial shift on two symbols i.e., there exists a set $\mathcal{B} \subset\{0,1\}^{\mathbb{Z}}$ that is invariant under the shift $\sigma:\{0,1\}^{\mathbb{Z}} \rightarrow\{0,1\}^{\mathbb{Z}}$ and a homeomorphism $\xi: \mathcal{B} \rightarrow \Lambda$

$$
\Psi \circ \xi=\xi \circ \sigma \text {. }
$$

Similar results are obtained in [36] in the case of the orbit-flip. Note that in general, the corresponding dynamics is not a subshift of finite type [17, 36].

The unfolding of degenerate homoclinic orbits can lead to even more complicated dynamics. A Hénon like attractor can be observed in the unfolding of a degenerate homoclinic orbit [25, 26], and Lorenz attractors can be observed after perturbation of a vector-field having a pair of homoclinic orbits [5, 35]. When studying a homoclinic orbit to a hyperbolic saddle, one often encounters the difficulty in estimating the Dulac map, that is the transition map between a section transverse to the local stable manifold to a section transverse to the unstable one. The presence of resonance has often been related with the lack of smoothness of the linearization near the singularity, and therefore making the estimation of the Dulac map more complicated. However, it can lead to increasing the complexity of the dynamics, see for instance [5, 24] for more details.

The paper is organized as follows. In the remainder of the introduction, we present a model of a twodimensional map on the plane which characterizes a re-injected horseshoe map. An example of an explicit map that satisfies the model properties can be found in [11]. We also state Theorem 1, the main result of the article, which realizes a re-injected horseshoe in Poincaré maps of a family of three-dimensional vector fields, and we give an outline of the steps in the proof. The proof of Theorem 1 is then given in Sections 2 and 3. In the final section we study an explicit map by choosing parameters for an approximate Poincaré map. We give a rigorous, computer-assisted characterization of the dynamics on the maximal invariant set of this map, which acts like a re-injected horseshoe. This characterization is through a semiconjugacy to a symbolic system. We then compare this to the dynamics of the corresponding cuspidal horseshoe alone, and the results strongly suggest that the dynamics of the full system has more complexity than that of the cuspidal horseshoe without re-injection.

\subsection{The model of the re-injected horseshoe map}

In the $(x, y)$-plane consider the domain

$$
S=S^{+} \cup S^{-} \text {where } S^{+}=(0,1] \times[-1,1] \text {, and } S^{-}=[-1 / 2,0) \times[-1,1] .
$$

The image of $S$ under $\Phi$ is described in Figure 2. The restriction of $\Phi$ to $S^{+}$is like a horseshoe except that one boundary is collapsed onto a cusp. The points that escape $S^{+}$are re-injected into $S^{+}$via the action of $\Phi$ on $S^{-}$. More precisely the model satisfies the following properties:

(i) $\Phi$ maps $S^{+}$and $S^{-}$diffeomorphically onto their respective images and $\Phi\left(S^{-}\right) \subset S^{+}$,

(ii) for each $y \in[-1,1], \Phi((0,1] \times\{y\})$ is a $C^{1}$ curve that intersects $W=\{0\} \times[-1,1]$ exactly twice,

(iii) there exists $0<x_{3}<x_{4}<1$ such that

$$
\Phi\left(\left\{x_{3}\right\} \times[-1,1]\right) \subset W, \Phi\left(\left\{x_{4}\right\} \times[-1,1]\right) \subset W
$$




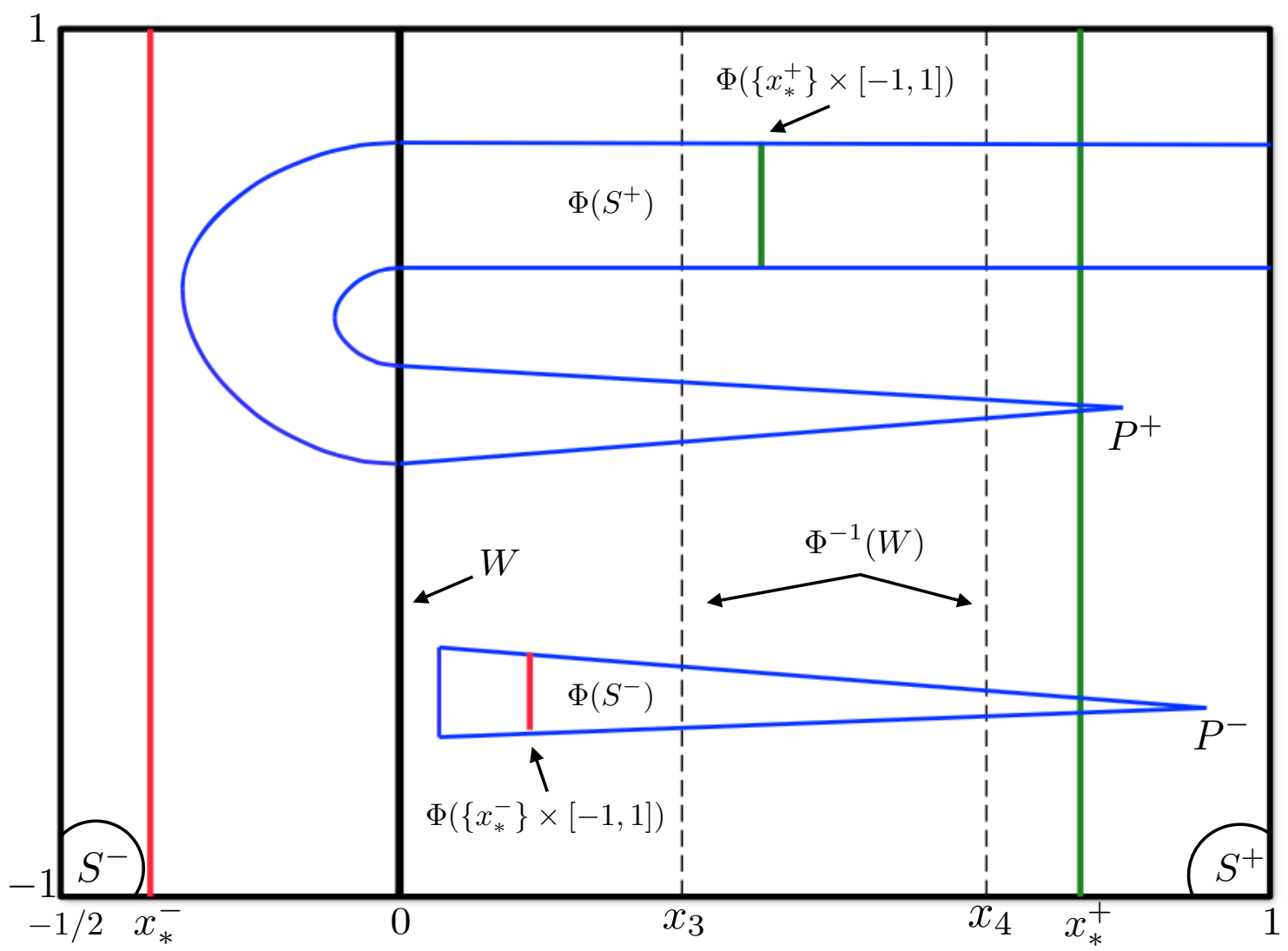

Figure 2: Description of the map $\Phi$.

and $\Phi(\{x\} \times[-1,1]) \cap W=\emptyset$ for all $x \neq x_{3}, x_{4}$,

(iv) for each $x \in[-1 / 2,0) \cup\left(0, x_{3}\right) \cup\left(x_{4}, 1\right]$, there exists $-1 / 2<L_{x}<1$ such that $\Phi(\{x\} \times[-1,1]) \subset$ $\left\{L_{x}\right\} \times[-1,1]$ so that vertical line segments form a $\Phi$-invariant foliation of $S^{+} \cup S^{-}$. Moreover, the map is expanding on this foliation, i.e. for any pair $x, x^{\prime}$ with $x \neq x^{\prime}$ which are both contained in $[-1 / 2,0)$, both contained in $\left(0, x_{3}\right)$, or both contained in $\left(x_{4}, 1\right]$, we have that

$$
\left|L_{x}-L_{x^{\prime}}\right|>\left|x-x^{\prime}\right|
$$

(v) there exist $P^{+}$and $P^{-}$in $S^{+}$such that $\lim _{x \rightarrow 0^{+}} \Phi(\{x\} \times[-1,1])=\left\{P^{+}\right\}, \lim _{x \rightarrow 0^{-}} \Phi(\{x\} \times[-1,1])=\left\{P^{-}\right\}$ where the limits are taken with respect to the Hausdorff distance,

(vi) $\Phi(\{1\} \times[-1,1]) \subset\{1\} \times[-1,1]$,

(vii) the restriction of $\Phi$ to $\Omega_{\mathbb{Z}}^{+}=\left\{P \in S^{+} \mid \Phi^{n}(P) \in S^{+}, \forall n \in \mathbb{Z}\right\}$ is hyperbolic.

The dynamics of such a model $\Phi$ depend on the specific map, in particular on the placement of the two cusps $P^{ \pm}$and the image $\Phi\left(S^{-}\right)$. On a $C^{1}$-open set of maps satisfying these properties, $\Omega_{\mathbb{Z}}^{+}$is a chaotic cuspidal horseshoe $[6,17,36,38]$. As $\Omega_{\mathbb{Z}}$ contains $\Omega_{\mathbb{Z}}^{+}$, the re-injected horseshoe dynamics is also chaotic. Figure 5(a) shows the images of the map $\varphi^{ \pm}$which is studied in Section 4 and is derived from an expansion of the Poincaré map of the class of vector fields described in the next section. Comparing the images to Figure 2, the orientations of the images are reversed. For $\varphi^{+}$the cusp $P^{+}$is above the rest of its image, and for $\varphi^{-}$the cusp is to the left of rest of its image. Also the image of $\varphi^{-}$lies inside the fold of the cuspidal horseshoe instead of below it as in Figure 2.

As indicated in the introduction, the first goal of this paper is to show that the dynamics of $\Phi$ on the set

$$
\Omega_{\mathbb{Z}}=\left\{P \in S \mid \Phi^{n}(P) \in S, \forall n \in \mathbb{Z}\right\}
$$


is realizable in the generic unfolding of a double homoclinic orbit in three-dimensional space, i.e. that the dynamics is $C^{1}$-conjugate to the Poincaré return map of a vector field family unfolding a degenerate homoclinic orbit. In the next section we describe how we retrieve that dynamics and state the main theorem of the article.

\subsection{A pair of homoclinic orbits}

Let $\mathcal{V} \subset \mathbb{R}^{3}$ be a neighborhood of the origin. Suppose $\mathcal{X}_{\gamma}$ is a generic, smooth $\left(C^{\infty}\right)$ family of vector fields on $\mathbb{R}^{3}$ for $\gamma \in \mathcal{V}$. We place hypotheses [H1]-[H5] on $\mathcal{X}_{\gamma}$ as follows.

[H1] for all $\gamma \in \mathcal{V}, \mathcal{X}_{\gamma}$ admits a hyperbolic equilibrium of saddle type.

By the Implicit Function Theorem, after a parameter dependent translation, one can assume that the singularity is fixed at the origin. We also assume that the eigenvalues $\{-\alpha(\gamma),-\beta(\gamma), \lambda(\gamma)\}$ of $d \mathcal{X}_{\gamma}(0)$ satisfy

[H2] $\alpha(\gamma)>0,2 \beta(\gamma) \equiv \lambda(\gamma)>0$, and $\alpha(\gamma)>\lambda(\gamma)$.

After a time rescaling, one can assume that $\lambda(\gamma) \equiv 1$ and $\mathcal{X}_{\gamma}$ takes the following form

$$
\mathcal{X}_{\gamma}(x, y, z)=x \frac{\partial}{\partial x}-\alpha(\gamma) y \frac{\partial}{\partial y}-\frac{z}{2} \frac{\partial}{\partial z}+\mathcal{R}(x, y, z)
$$

where $\alpha(\gamma)>1$ and $\mathcal{R}$ stands for the nonlinear terms.

Thus, at the origin $\mathcal{X}_{\gamma}$ admits a two-dimensional local stable manifold $W_{\text {loc }}^{s}$ and one-dimensional local unstable manifold $W_{\text {loc }}^{u}$. We extend $W_{\text {loc }}^{s}$ by the backward iteration of the flow and obtain the global stable manifold $W^{s}$. The local strong stable manifold $W_{\text {loc }}^{s s} \subset W_{\text {loc }}^{s}$ has its tangent space at the origin spanned by the eigenspace associated to $-\alpha(\gamma)$. These manifolds are invariant, smooth, and unique. Furthermore, there exists a local invariant manifold which is tangent at the origin to the eigenspace associated with the eigenvalues 1 and $-\beta$. This manifold is denoted by $W_{\text {loc }}^{s, u}$ and is called an extended unstable manifold. Such an invariant set contains $W_{\mathrm{loc}}^{u}$, is not unique, and is $C^{k}$ where $k$ is the integer part of $\alpha / \beta>2$. However its tangent space along the local unstable manifold $W_{\text {loc }}^{u}$ is unique. See [15] for more details. Observe that the flow starting from a point on the stable manifold accumulates to the origin, therefore the stable manifold acts as a separatrix. In this setting we have

$$
W_{\mathrm{loc}}^{s} \subset\{x=0\}, W_{\mathrm{loc}}^{s s} \subset\{x=0=z\}, W_{\mathrm{loc}}^{u} \subset\{z=0=y\},
$$

and we can choose $W_{\text {loc }}^{u, s} \subset\{y=0\}$.

We further assume that

[H3] $\mathcal{X}_{0}$ admits two homoclinic orbits to the origin, $\Gamma_{1}=\left\{\Gamma_{1}(t) \mid t \in \mathbb{R}\right\}$ and $\Gamma_{2}=\left\{\Gamma_{2}(t) \mid t \in \mathbb{R}\right\}$, and that $\Gamma_{1}$ is an inclination-flip homoclinic orbit, that is, the global stable manifold $W^{s}$ intersects any extended unstable manifold along $\Gamma_{1}$ in a tangency of quadratic contact.

[H4] At the same time we assume that $\Gamma_{2}$ is a nondegenerate homoclinic orbit, i.e., the global stable manifold $W^{s}$ intersects any extended unstable manifold along $\Gamma_{2}$ transversally, and $W_{\text {loc }}^{s s} \cap \Gamma_{2}=\{0\}$.

Observe that the latter assumption amounts to assuming that $\Gamma_{2}$ is not of orbit-flip type. See [5, 6, 17, 21, $25,38]$ for more details and discussions.

In the three-dimensional context, a double homoclinic loop forms either a figure-eight shape or a butterfly shape. In this article we assume that

[H5] $\Gamma_{1} \cup \Gamma_{2}$ forms a butterfly shape, that is, $W_{\text {loc }}^{s s}$ splits $W_{\text {loc }}^{s}$ into two connected components, say $W_{+}^{s}=$ $\left\{(x, y, z) \in W_{\mathrm{loc}}^{s} \mid z>0\right\}$ and $W_{-}^{s}=\left\{(x, y, z) \in W_{\mathrm{loc}}^{s} \mid z<0\right\}$, and $\Gamma_{1}(t) \cup \Gamma_{2}(t) \subset W_{+}^{s}$ for $t$ large enough; see Figure 3.

Proposition 1 Let $\hat{S}$ be a two-dimensional section that is transverse to $W_{\mathrm{loc}}^{s}$. Let $(x, y)$ be a smooth parametrization of $\hat{S}$ such that

$$
\{x=0\} \cap \hat{S}=W_{\text {loc }}^{s}, \text { and } \Gamma_{1}(t) \subset\{x>0\} \text { for large } t .
$$




$$
\phi: \hat{S} \cap\{x>0\} \rightarrow \hat{S},(x, y) \mapsto\left(\phi_{1}(x, y), \phi_{2}(x, y)\right)
$$

be the Poincaré return map associated to $\mathcal{X}_{0}$, the unperturbed system. Then

$$
c_{M}:=\lim _{x \rightarrow 0^{+}} \frac{\partial \phi_{1}(x, y)}{\partial x}
$$

does not depend on the choice of the section $\hat{S}$ nor the choice of the parameterization.

The proof of this proposition is at the end of Section 2. The intrinsic quantity $c_{M}$ is called the Melnikov exponent of the Poincaré return map. We are now in position to state the main theorem of this article.

Theorem 1 Suppose $\mathcal{X}_{\gamma}$ is a generic family of smooth $\left(C^{\infty}\right)$ vector fields satisfying hypotheses [H1]-[H5] above. We further assume that the Melnikov exponent satisfies $1<c_{M}<4$. Then there exists an open set $\mathcal{V}^{*} \subset \mathcal{V}$ such that for all $\gamma \in \mathcal{V}^{*}, \mathcal{X}_{\gamma}$ admits a suspended re-injected cuspidal horseshoe.

Due to the re-injection, the suspended re-injected horseshoe together with the origin and connections along the stable manifold of the origin is an attractor in the three-dimensional flow, in a similar manner as the Lorenz attractor. Indeed we construct a Poincaré map on a section $S$ which maps all points in $S$ strictly into $S$ or into the stable manifold of the origin. The set of points which do not map to the stable manifold of the origin after finitely many iterations is the re-injected horseshoe.

The proof of Theorem 1 proceeds as follows. In Section 2 we compute the Poincaré return map associated to $\mathcal{X}_{\gamma}$ on a given section. This return map is the composition of a local map near the equilibrium point and a map for the excursion away from the equilibrium point which admits a regular series expansion. Since the vector field has a persistent resonance at the origin, we cannot assume that it is smoothly linearizable, and hence the expansion of the local map must be expressed on a Dulac scale. In Section 3, the composition of these expansions is studied, and we show that there is a narrow region of parameter space for which the Poincaré map on an appropriate portion of the section behaves geometrically like a re-injected horseshoe (Sections 3.1-3.3). A blow-up in the parameter space is required to obtain the right amount of expansion in the image of the map (Section 3.4). Finally, we show that the resulting map has an invariant foliation conjugate to that described in Section 1.1 by proving that the map on the cuspidal horseshoe without re-injection admits a hyperbolic structure via an invariant cone field (Section 3.5).

\section{Computing the Poincaré map}

The Poincaré map is the composition of a local map near the equilibrium, which admits a Dulac expansion (see below), and a regular map. Let $S$ be a two-dimensional section transverse to the local stable manifold, and let $\Sigma^{+}, \Sigma^{-}$be two-dimensional sections, each transverse to a branch of the unstable manifold, as indicated in Figure 3.

\subsection{The regular map}

After some rescaling, we choose the sections to be

$$
\begin{gathered}
S=\left\{(x, y, z) \in \mathbb{R}^{3}|z=1,| x\left|\leq \delta_{1},\right| y \mid<1\right\}, \\
\Sigma^{ \pm}=\left\{(x, y, z) \in \mathbb{R}^{3} \mid x= \pm 1, \max \{|y|,|z|\} \leq \delta_{2}\right\},
\end{gathered}
$$

where $\delta_{1}>0$ and $\delta_{2}>0$ are sufficiently small so that the corresponding Poincaré transition maps

$$
\begin{array}{ll}
\phi_{\text {reg }}^{+}: \Sigma^{+} \rightarrow \mathbb{R}^{3} & \text { given by } \quad(1, Y, Z) \mapsto\left(\phi_{\text {reg }, 1}^{+}(Y, Z), \phi_{\text {reg }, 2}^{+}(Y, Z), 1\right) \text { and } \\
\phi_{\text {reg }}^{-}: \Sigma^{-} \rightarrow \mathbb{R}^{3} & \text { given by } \quad(-1, Y, Z) \mapsto\left(\phi_{\text {reg }, 1}^{-}(Y, Z), \phi_{\text {reg }, 2}^{-}(Y, Z), 1\right)
\end{array}
$$



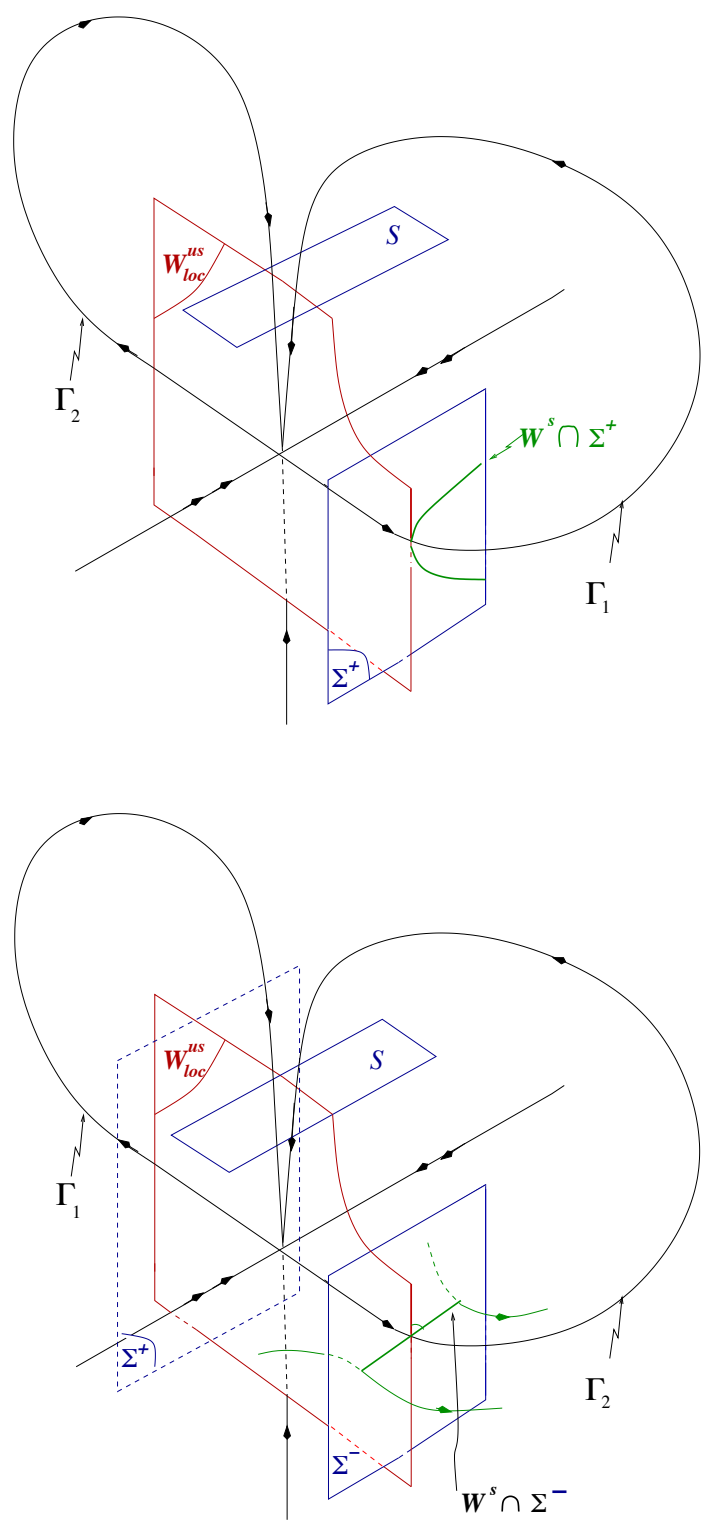

Figure 3: Phase portrait of $\mathcal{X}_{0}$. The top figure emphasizes $\Gamma_{1}$ and the quadratic tangency between $W^{s}$ and $W_{\mathrm{loc}}^{u, s}$. The bottom figure is obtained from that on the top by a rotation about the $z$-axis and emphasizes $\Gamma_{2}$. 
are well defined. Furthermore, $\phi_{\mathrm{reg}, 1}^{ \pm}(Y, Z)$ and $\phi_{\mathrm{reg}, 2}^{ \pm}(Y, Z)$ admit the following expansions

$$
\begin{aligned}
& \phi_{\mathrm{reg}, 1}^{+}(Y, Z)=\varepsilon_{1}+a_{1}^{+} Y-\mu Z+\text { h.o.t. }{ }_{1}^{+}(Y, Z) \\
& \phi_{\mathrm{reg}, 2}^{+}(Y, Z)=\omega_{1}+a_{2}^{+} Y+b_{2}^{+} Z+\text { h.o.t.2 } 2(Y, Z) \\
& \phi_{\mathrm{reg}, 1}^{-}(Y, Z)=\varepsilon_{2}+a_{1}^{-} Y+b_{1}^{-} Z+\text { h.o.t. } ._{1}^{-}(Y, Z) \\
& \phi_{\mathrm{reg}, 2}^{-}(Y, Z)=\omega_{2}+a_{2}^{-} Y+b_{2}^{-} Z+\text { h.o.t. }{ }_{2}^{-}(Y, Z),
\end{aligned}
$$

where h.o.t. ${ }_{1,2}^{ \pm}$denotes higher order nonlinear terms. In particular we emphasize

$$
\text { h.o.t. }{ }_{1}^{+}(Y, Z)=c Z^{2}+c_{1} Z^{3}+Z^{4} \mathcal{R}_{1}(Z)+Y \mathcal{R}_{2}(Y, Z) .
$$

Recall that each parameter we introduce depends on $\gamma \in \mathcal{V} \subset \mathbb{R}^{3}$. However, we do not emphasize the $\gamma$-dependence when there is no confusion. In particular we have

$$
\begin{gathered}
\varepsilon_{1}=\varepsilon_{1}(\gamma)=\phi_{\mathrm{reg}, 1}^{+}(0,0), \varepsilon_{2}=\varepsilon_{2}(\gamma)=\phi_{\mathrm{reg}, 1}^{-}(0,0), \\
\mu=\mu(\gamma)=-\frac{\partial \phi_{\mathrm{reg}, 1}^{+}}{\partial Z}(0,0), b_{1}^{-}=b_{1}^{-}(\gamma)=\frac{\partial \phi_{\mathrm{reg}, 1}^{-}}{\partial Z}(0,0) .
\end{gathered}
$$

In this setting, $\mathcal{X}_{0}$ admits $\Gamma_{1}$ as an inclination-flip homoclinic orbit if and only if $\varepsilon_{1}(0)=0=\mu(0)$, and $\Gamma_{2}$ is a nondegenerate homoclinic orbit if and only if $\varepsilon_{2}(0)=0$ but $b_{1}^{-}(0) \neq 0$, say

$$
b_{1}^{-}=b_{1}^{-}(\gamma)<0
$$

For the unperturbed system, $W^{s}$ and $W_{\text {loc }}^{u, s}$ have a tangency of quadratic contact, which further implies that

$$
\left.\frac{\partial^{2} \phi_{\text {reg }, 1}}{\partial Z^{2}}\right|_{\gamma=0}=\left.c(\gamma)\right|_{\gamma=0} \neq 0 \text {, say } c(0)>0 \text {. }
$$

Finally, by genericity of the family $\mathcal{X}_{\gamma}$, we can assume the map

$$
\gamma \mapsto\left(\varepsilon_{1}(\gamma), \varepsilon_{2}(\gamma), \mu(\gamma)\right)
$$

is a diffeomorphism near the origin, and we identify $\gamma$ with $\left(\varepsilon_{1}, \varepsilon_{2}, \mu\right)$.

\subsection{The local map and its expansion}

Since the vector field at the origin admits a persistent resonance, we cannot assume the vector field to be $C^{r}$-linearizable for large $r$. This implies that we need to have information on the asymptotics of the local map, often called the Dulac map. For simplicity, we shall assume that $\beta=1 / 2$ is the unique resonance for the linearization of $\mathcal{X}_{0}$, which occurs if and only if both $\alpha$ and $\alpha / \beta$ are irrational. In the present context, such a map admits a Dulac expansion. More precisely, we say that $g: \mathbb{R} \rightarrow \mathbb{R}$, admits a Dulac expansion if $g(x)$ expands in the Dulac scale

$$
\left\{1, x \log |x|, x, x^{2} \log ^{2}|x|, x^{2} \log |x|, x^{2}, \ldots x^{k} \log ^{k}|x|, x^{k} \log ^{k-1}|x|, \cdots x^{k}, \cdots\right\},
$$

that is,

$$
\begin{aligned}
g(x) & =p_{0}+p_{1} x \log |x|+p_{2} x+p_{3} x^{2} \log ^{2}|x|+\cdots \\
& +p_{m} x^{k} \log ^{k}|x|+\cdots+p_{m+k} x^{k}+\cdots
\end{aligned}
$$

for some unique real coefficients $p_{k}$. For such an expansion we write

$$
g(x)=\hat{O}\left(x^{k} \log ^{\ell}(x)\right)
$$

if the coefficient associated to $x^{k} \log ^{\ell}(x)$ is the first nonzero term in the expansion. Also, if the coefficient depends smoothly on $y$, we write

$$
g(x, y)=\hat{O}_{y}\left(x^{k} \log ^{\ell}(x)\right) .
$$


Observe that we prefer to write $\hat{O}\left(x^{0}\right)$ instead of $\hat{O}(1)$ to emphasize that the corresponding expansion concerns the variable $x$. The coefficients may depend on the parameter $\gamma$ but we suppress this dependence unless it is necessary for clarity.

Define

$$
S^{+}=\{(x, y, 1) \in S \mid x>0\} \text { and } S^{-}=\{(x, y, 1) \in S \mid x<0\} .
$$

We state the following lemma.

Lemma 1 The local map $\phi_{\mathrm{loc}}^{ \pm}: S^{ \pm} \rightarrow \Sigma^{ \pm}$given by

$$
(x, y, 1) \mapsto\left( \pm 1, \phi_{\mathrm{loc}, 2}^{ \pm}(x, y), \phi_{\mathrm{loc}, 3}^{ \pm}(x, y)\right)
$$

takes the form

$$
\left\{\begin{array}{l}
\phi_{\mathrm{loc}, 2}^{ \pm}(x, y)=|x|^{\alpha} y\left(1+Q_{1}^{ \pm}(x)\right) \\
\phi_{\mathrm{loc}, 3}^{ \pm}(x, y)=\sqrt{|x|}\left(1+Q_{2}^{ \pm}(x)\right),
\end{array}\right.
$$

up to a $C^{\infty}$ change of coordinates, where $Q_{i}^{ \pm}$for $i=1,2$ admits the following expansion

$$
Q_{i}^{ \pm}(x)=p_{1, i}|x| \log |x|+p_{2, i} x+p_{3, i}|x|^{2} \log ^{2}|x|+\cdots=\hat{O}(|x| \log |x|)
$$

where the coefficients $p_{j, i}$ depend smoothly on $\gamma$.

The proof of this lemma follows directly from [4], see also [5, 34].

\subsection{The Melnikov exponent}

The Poincaré return map

$$
\phi=\phi_{\gamma}: S^{+} \cup S^{-} \rightarrow S,
$$

defined as the composition of the local and regular maps,

$$
\phi=\phi_{\mathrm{reg}} \circ \phi_{\mathrm{loc}},
$$

has the form

$$
\phi(x, y, 1)=\left\{\begin{array}{lll}
\left(\phi_{1}^{+}(x, y), \phi_{2}^{+}(x, y), 1\right) & \text { if } \quad x>0, \\
\left(\phi_{1}^{-}(x, y), \phi_{2}^{-}(x, y), 1\right) & \text { if } \quad x<0 .
\end{array}\right.
$$

From equations (1) and (2) and Lemma 1, if

$$
Y=|x|^{\alpha} y\left(1+Q_{1}^{ \pm}(x)\right), \quad Z=|x|^{1 / 2}\left(1+Q_{2}^{ \pm}(x)\right),
$$

then it follows that for all integers $m>0$ and $n>0$ we have

$$
Y^{m} Z^{n}=|x|^{n \alpha+m / 2} y^{m}\left(1+Q_{3, m, n}^{ \pm}(x)\right)
$$

where $Q_{3, m, n}^{ \pm}(x)$ admits the same type of asymptotics as $Q_{1,2}^{ \pm}(x)$, so that

$$
Q_{3, m, n}^{ \pm}(x)=\hat{O}(|x| \log (x)) .
$$

As a consequence, the Poincaré map takes the form

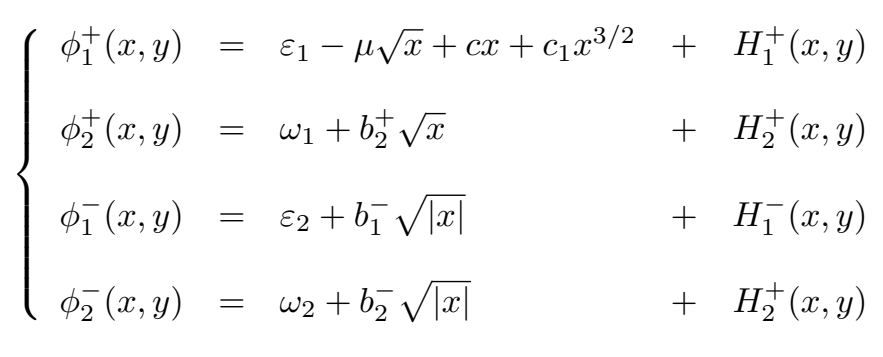


and using the above Landau notation we have

$$
\left\{\begin{aligned}
H_{1}^{+}(x, y) & =x \hat{O}(x \log (x))+\mu x^{1 / 2} \hat{O}(x \log (x))+x^{\alpha} y \hat{O}_{y}\left(x^{0}\right) \\
H_{2}^{+}(x, y) & =\hat{O}(x)+x^{\alpha} y \hat{O}_{y}\left(x^{0}\right) \\
H_{1,2}^{-}(x, y) & =\hat{O}(x)+|x|^{\alpha} y \hat{O}_{y}\left(x^{0}\right) .
\end{aligned}\right.
$$

Also note that without loss of generality, we can rescale and choose the sections so that

$$
\phi_{2}^{+}\left(S^{+}\right) \subset[-1 / 2,1 / 2] .
$$

In particular, from equations (5) and (6) for the unperturbed system, $\phi^{+}$takes the form

$$
\left\{\begin{array}{l}
\phi_{1}^{+}(x, y)=c x+c_{1} x^{3 / 2}+x \hat{O}(x \log (x))+x^{\alpha} y \hat{O}_{y}\left(x^{0}\right) \\
\phi_{2}^{+}(x, y)=\omega_{1}+b_{2}^{+} \sqrt{x}+\hat{O}(x)+x^{\alpha} y \hat{O}_{y}\left(x^{0}\right)
\end{array}\right.
$$

Now let $\tau_{\omega_{1}}: S \rightarrow S$ be given by

$$
(x, y, 1) \mapsto\left(x, y+\omega_{1}, 1\right)
$$

and write

$$
\tau_{\omega_{1}}^{-1} \circ \phi^{+} \circ \tau_{\omega_{1}}(x, y, 1)=\left(\varphi_{1}(x, y), \varphi_{2}(x, y), 1\right) .
$$

Then the map (8) is conjugated to the map

$$
\varphi(x, y)=\left(\varphi_{1}(x, y), \varphi_{2}(x, y)\right)
$$

where

$$
\begin{aligned}
& \varphi_{1}(x, y)=c x+\sqrt{x} \hat{O}(x)+x^{\alpha} y \hat{O}_{y}\left(x^{0}\right) \quad \text { and } \\
& \varphi_{2}(x, y)=b_{2}^{+} \sqrt{x}+\hat{O}(x)+x^{\alpha} y \hat{O}_{y}\left(x^{0}\right) .
\end{aligned}
$$

Consider now the group $\mathcal{G}$ of germs of smooth $\left(C^{\infty}\right)$ diffeomorphisms $F:\left(\mathbb{R}^{2}, 0\right) \rightarrow\left(\mathbb{R}^{2}, 0\right)$ of the form

$$
(x, y) \mapsto\left(F_{1}(x, y), F_{2}(x, y)\right)
$$

that map $\{x=0\}$ onto itself and map $\mathbb{R}_{+} \times \mathbb{R}$ onto itself. It follows that

$$
F_{1}(x, y)=x \widehat{F}_{1}(x, y)
$$

where $\widehat{F}_{1}$ is smooth, and positive whenever $x$ is positive. For each $F \in \mathcal{G}$, we define $\Psi_{F}(\varphi):\left(\mathbb{R}^{2}, 0\right) \mapsto\left(\mathbb{R}^{2}, 0\right)$ by

$$
(x, y) \mapsto\left(F \circ \varphi \circ F^{-1}(x, y)\right)=\left(\Psi_{F}(\varphi)_{1}(x, y), \Psi_{F}(\varphi)_{2}(x, y)\right) .
$$

Lemma 2 For all $F \in \mathcal{G}$

$$
\left.\frac{\partial}{\partial x} \Psi_{F}(\varphi)_{1}(x, y)\right|_{x=0}=c
$$

Proof: Let $F \in \mathcal{G}$ and write the Taylor expansion of $F$ near $(0,0)$. Observe that even if there are no logarithmic terms, we still can use the Landau notation above to obtain

$$
F(x, y)=\left(L_{11} x+x^{2} \hat{O}_{y}\left(x^{0}\right)+x y \hat{O}_{y}\left(x^{0}\right), L_{21} x+L_{22} y+y \hat{O}_{y}\left(x^{0}\right)+x^{2} \hat{O}_{y}\left(x^{0}\right)\right)
$$

where $L_{11}>0$ and $L_{22} \neq 0$. For $G=F^{-1}$ we have

$$
\begin{aligned}
G(x, y)= & \left(\frac{x}{L_{11}}+x^{2} \hat{O}_{y}\left(x^{0}\right)+x y \hat{O}_{y}\left(x^{0}\right),\right. \\
& \left.-\frac{L_{21}}{L_{22} L_{11}} x+\frac{y}{L_{22}}+x^{2} \hat{O}_{y}\left(x^{0}\right)+x y \hat{O}_{y}\left(x^{0}\right)\right)
\end{aligned}
$$


From the above, we have that

$$
\begin{aligned}
\varphi_{1} \circ G(x, y) & =\frac{c}{L_{11}} x+x y \hat{O}_{y}\left(x^{0}\right)+x^{1 / 2} \hat{O}_{y}(x) \\
\varphi_{2} \circ G(x, y) & =b_{2}^{+}\left(\frac{x}{L_{11}}+x^{2} \hat{O}_{y}\left(x^{0}\right)+x y \hat{O}_{y}\left(x^{0}\right)\right)^{1 / 2} \\
& =\frac{b_{2}^{+}}{L_{11}} \sqrt{x}+x^{1 / 2} \hat{O}_{y}(x) .
\end{aligned}
$$

Finally $\Psi_{F}(\varphi)_{1}$ takes the form

$$
\Psi_{F}(\varphi)_{1}(x, y)=c x+x y \hat{O}_{y}\left(x^{0}\right)+x^{1 / 2} \hat{O}_{y}\left(x^{0}\right)
$$

and Lemma 2 follows.

Proof of Proposition 1: Let us consider two sections $\underset{\tilde{S}}{S}$ and $\tilde{S}$ transverse to $W^{u}$ and consider the holonomy map $h: S \rightarrow \tilde{S}$ associated to $\mathcal{X}_{0}$. We parametrize $\tilde{S}$ with $(\tilde{x}, \tilde{y})$ such that

$$
\{\tilde{x}=0\} \cap \tilde{S}=\tilde{S} \cap W_{\text {loc }}^{s} .
$$

Therefore

$$
h(\{x=0\}) \subset\{\tilde{x}=0\} .
$$

We can also assume that

$$
h(\{x>0\} \cap S) \subset\{\tilde{x}>0\} \text { and }\{\tilde{x}=0 \cap \tilde{y}=0\}=W^{u} \cap \tilde{S} .
$$

This implies that $h \in \mathcal{G}$.

As shown above, the Poincaré map $\phi$ on $S$ for the unperturbed system is conjugate to $\varphi$. Hence the Poincaré return map computed on $\tilde{S}$ for the unperturbed system is conjugate to $\Psi_{h}(\varphi)=h \circ \varphi \circ h^{-1}$. Lemma 2 implies these two maps have the same linear coefficient, the Melnikov exponent.

Note that the coefficient $c$ in the Poincaré map $\phi$ given in (5) is the Melnikov exponent $c_{M}$.

\section{The Poincaré return map}

We first study the Poincaré return map restricted to the section $S^{+}$i.e.,

$$
(x, y) \mapsto\left(\phi_{1}^{+}(x, y), \phi_{2}^{+}(x, y)\right) .
$$

We begin by studying the folded region included in $S^{-}$. We then fix $y \in[-1,1]$ and look at the curve

$$
\mathbf{C}_{y}=\left\{\left(\phi_{1}^{+}(x, y), \phi_{2}^{+}(x, y)\right), x>0\right\} .
$$

To show that this curve is as like in Figure 4 , we fix $\varepsilon_{1}, \mu$ and look at a critical point $x_{c}=x_{c}(y, \mu)$ such that

$$
\frac{\partial \phi_{1}^{+}}{\partial x}\left(x_{c}(y, \mu), y\right) \equiv 0
$$

\subsection{The folded region}

From (5) and (6), we have

$$
\frac{\partial \phi_{1}^{+}}{\partial x}(x, y)=-\frac{\mu}{2 \sqrt{x}}+c+\frac{3}{2} c_{1} \sqrt{x}+\frac{\partial H_{1}^{+}}{\partial x}(x, y)
$$

i.e., $x_{c}$ satisfies

$$
\mu / 2=c \sqrt{x_{c}}+\frac{3}{2} c_{1} x_{c}+\sqrt{x_{c}} \frac{\partial H_{1}^{+}}{\partial x}\left(x_{c}, y\right)
$$




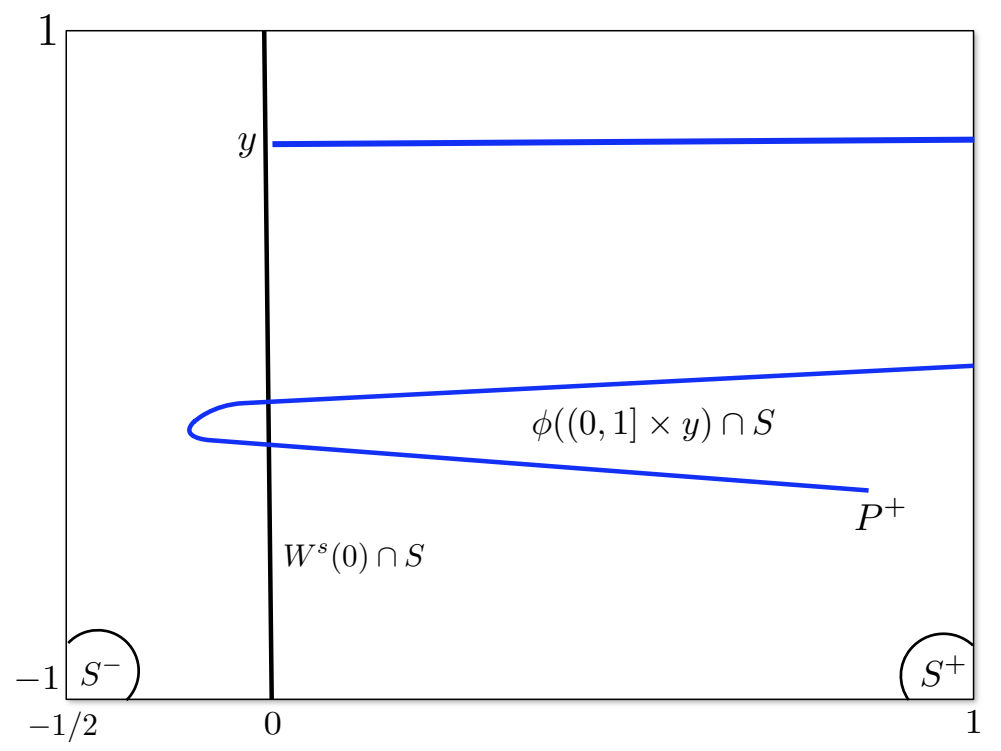

Figure 4: Folded images of $\phi^{+}$.

From (6) we have that

$$
\frac{\partial H_{1}^{+}}{\partial x}(x, y)=\hat{O}(x \log (x))+x^{-1 / 2} \mu \hat{O}(x \log |x|)+x^{\alpha-1} y \hat{O}_{y}\left(x^{0}\right) .
$$

We introduce $q_{c}=\sqrt{x_{c}}$, with (9) and (10) we have

$$
\mu / 2=c q_{c}+\frac{3}{2} c_{1} q_{c}^{2}+q_{c} \frac{\partial H_{1}^{+}}{\partial x}\left(q_{c}^{2}, y\right)
$$

where

$$
q_{c} \frac{\partial H_{1}^{+}}{\partial x}\left(q_{c}^{2}, y\right)=\hat{O}\left(q_{c}^{3} \log \left(q_{c}\right)\right)+\mu \hat{O}\left(q_{c}^{2} \log \left(q_{c}\right)\right)+q_{c}^{2 \alpha-1} y \hat{O}_{y}\left(q_{c}^{0}\right)
$$

Lemma 3 There exists a smooth function $H_{3}$ defined near the origin in $\mathbb{R}^{2}$ such that if (11) holds then

$$
q_{c}=q_{c}(y, \mu)=\frac{\mu}{2 c}-\frac{3 c_{1} \mu^{2}}{8 c^{3}}+H_{3}(y, \mu)
$$

where

$$
H_{3}(y, \mu)=\hat{O}\left(\mu^{3} \log |\mu|\right)+y \mu^{2 \alpha-1} \hat{O}_{y}\left(\mu^{0}\right)
$$

Proof: From (11) and (12) we have

$$
\frac{\mu}{2 c}+\mu \hat{O}\left(q_{c}^{2} \log \left(q_{c}\right)\right)=q_{c}+\frac{3}{2 c} c_{1} q_{c}^{2}+y q_{c}^{2 \alpha-1} \hat{O}_{y}\left(q_{c}^{0}\right)+\hat{O}\left(q_{c}^{3} \log \left(q_{c}\right)\right)
$$

i.e.,

$$
\begin{aligned}
\frac{\mu}{2 c}\left(1+\hat{O}\left(q_{c}^{2} \log \left(q_{c}\right)\right)\right) & =q_{c}\left(1+\frac{3}{2 c} c_{1} q_{c}+\hat{O}\left(q_{c}^{2} \log \left(q_{c}\right)\right.\right. \\
& \left.+y q_{c}^{2 \alpha-2} \hat{O}_{y}\left(q_{c}^{0}\right)\right)
\end{aligned}
$$


and therefore

$$
\frac{\mu}{2 c}=q_{c} \frac{\left(1+\frac{3}{2 c} c_{1} q_{c}+\hat{O}\left(q_{c}^{2} \log \left(q_{c}\right)+q_{c}^{2 \alpha-2} y \hat{O}_{y}\left(q_{c}^{0}\right)\right)\right.}{\left(1+\hat{O}\left(q_{c}^{2} \log \left(q_{c}\right)\right)\right)} .
$$

Using $(1-w)^{-1}=1+w+w^{2}+\cdots,(13)$ can be written

$$
\left.\frac{\mu}{2 c}=q_{c}\left(1+\frac{3}{2 c} c_{1} q_{c}+\hat{O}\left(q_{c}^{2} \log \left(q_{c}\right)\right)+q_{c}^{2 \alpha-2} y \hat{O}_{y}\left(q_{c}^{0}\right)\right)\right) .
$$

Let by $X=\log (\mu /(2 c))$ and $Y=\log \left(q_{c}\right)$. Applying the logarithmic function on both sides of (14) and using the fact that $\log (1+w)=w-w^{2} / 2+w^{3} / 3+\cdots$, we have

$$
X=Y+\frac{3}{2 c} c_{1} q_{c}+\hat{O}\left(q_{c}^{2} Y\right)+q_{c}^{2 \alpha-2} y \hat{O}_{y}\left(q_{c}^{0}\right) .
$$

The above expression is indeed a formal expansion in $X$ and $Y$ without logarithmic term. Also, using the fact that

$$
(1+w)^{2 \alpha-2}=1+(2 \alpha-2) w+\cdots,
$$

by setting $Q_{\alpha}=q_{c}^{2 \alpha-2}$ and $M_{\alpha}=\mu^{2 \alpha-2}$, from (14), we have

$$
\left.M_{\alpha}=(2 c)^{2 \alpha-2} Q_{\alpha}\left(1+(2 \alpha-2) \frac{3}{2 c} c_{1} q_{c}+\hat{O}\left(q_{c}^{2} \log \left(q_{c}\right)\right)+Q_{c} y \hat{O}_{y}\left(q_{c}^{0}\right)\right)\right) .
$$

Equations (13), (15) and (16) take the form

$$
\left(\frac{\mu}{2 c}, X, M_{\alpha}\right)=\mathbf{G}\left(q_{c}, Y, Q_{\alpha}, y\right)
$$

where $\mathbf{G}$ is a smooth function and

$$
\left(\frac{\partial \mathbf{G}}{\partial q_{c}}, \frac{\partial \mathbf{G}}{\partial Y}, \frac{\partial \mathbf{G}}{\partial Q_{\alpha}}\right)
$$

has full rank near $(0,0,0)$. By the Implicit Function Theorem there exists $\mathbf{H}$ such that

$$
\left(q_{c}, Y, Q_{\alpha}\right)=\mathbf{H}\left(\frac{\mu}{2 c}, X, M_{\alpha}, y\right)
$$

which is also smooth. From (13), (15) and (16) we have

$$
\left\{\begin{aligned}
q_{c} & =\frac{\mu}{2 c}-\frac{3}{2 c} c_{1} \frac{\mu^{2}}{4 c^{2}}+\hat{O}\left(\mu^{3} X\right)+y \mu M_{\alpha} \hat{O}_{y}\left(\mu^{0}\right) \\
Y & =X-\frac{3 \mu}{4 c^{2}} c_{1}+\hat{O}\left(\mu^{2} X\right)++y M_{\alpha} \hat{O}_{y}\left(\mu^{0}\right) \\
Q_{\alpha} & \left.=\frac{M_{\alpha}}{(2 c)^{2 \alpha-2}}\left(1+(2 \alpha-2) 3 c_{1} \mu+\hat{O}\left(\mu^{2} X\right)+M_{\alpha} y \hat{O}_{y}\left(\mu^{0}\right)\right)\right),
\end{aligned}\right.
$$

and therefore we have

$$
q_{c}=\frac{\mu}{2 c}-\frac{3 \mu^{2}}{8 c^{3}} c_{1}+\hat{O}\left(\mu^{3} \log |\mu|\right)+y \mu^{2 \alpha-1} \hat{O}_{y}\left(\mu^{0}\right)
$$

ending the proof of the lemma.

By Lemma 3, we have

$$
q_{c}=\frac{\mu}{2 c}\left(1-\frac{3 c_{1} \mu}{4 c^{2}}+\frac{2 c}{\mu} H_{3}(y, \mu)\right)
$$

and therefore

$$
q_{c}^{2}=x_{c}=\frac{\mu^{2}}{4 c^{2}}\left(1-\frac{3 c_{1} \mu}{2 c^{2}}+H_{4}(y, \mu)\right)=\frac{\mu^{2}}{4 c^{2}}-\frac{3 c_{1} \mu^{3}}{8 c^{4}}+H_{5}(y, \mu)
$$


where

$$
\begin{aligned}
& H_{4}(y, \mu)=\hat{O}\left(\mu^{2} \log \mu\right)+y \mu^{2 \alpha-2} \hat{O}\left(\mu^{0}\right) \\
& H_{5}(y, \mu)=\hat{O}\left(\mu^{4} \log \mu\right)+y \mu^{2 \alpha} \hat{O}\left(\mu^{0}\right) .
\end{aligned}
$$

We also obtain

$$
\begin{aligned}
q_{c}^{3}=x_{c}^{3 / 2} & =\frac{\mu^{3}}{8 c^{3}}\left(1-\frac{3 c_{1} \mu}{2 c^{2}}+H_{4}(y, \mu)\right)^{3 / 2} \\
& =\frac{\mu^{3}}{8 c^{3}}+H_{6}(y, \mu)
\end{aligned}
$$

where

$$
H_{6}(y, \mu)=\hat{O}\left(\mu^{4} \log \mu\right)+y \mu^{2 \alpha+1} \hat{O}\left(\mu^{0}\right) .
$$

We now compute the corresponding critical value i.e.,

$$
\begin{aligned}
\phi_{1}^{+}\left(x_{c}(y, \mu), y\right) & =V\left(\varepsilon_{1}, \mu, y\right) \\
& =\varepsilon_{1}-\mu \sqrt{x_{c}}+c x_{c}+c_{1} x_{c}^{3 / 2}+H_{1}^{+}\left(x_{c}(y, \mu), y\right) .
\end{aligned}
$$

By (6) we have that

$$
H_{1}^{+}\left(x_{c}(y, \mu), y\right)=\mu^{2} \hat{O}\left(\mu^{2} \log \mu\right)+y \mu^{2 \alpha} \hat{O}\left(\mu^{0}\right),
$$

and the above calculations lead to

$$
\begin{aligned}
V\left(\varepsilon_{1}, \mu, y\right) & =\varepsilon_{1}-\frac{\mu^{2}}{2 c}+\frac{3 c_{1} \mu^{3}}{8 c^{3}}-\mu H_{3}(y, \mu)+\frac{\mu^{2}}{4 c}-\frac{3 c_{1} \mu^{3}}{8 c^{3}}+c_{1} \frac{\mu^{3}}{8 c^{3}} \\
& +c H_{5}(y, \mu)+c_{1} H_{6}(y, \mu)+H_{1}^{+}\left(x_{c}(\mu, y), y\right) \\
& =\varepsilon_{1}-\frac{\mu^{2}}{4 c}+c_{1} \frac{\mu^{3}}{8 c^{3}}+G_{3}(\mu, y)
\end{aligned}
$$

where

$$
\begin{aligned}
G_{3}(\mu, y) & =-\mu H_{3}(y, \mu)+c H_{5}(y, \mu)+c_{1} H_{6}(y, \mu)+H_{1}^{+}\left(x_{c}(y, \mu), y\right) \\
& =\hat{O}\left(\mu^{4} \log \mu\right)+y \mu^{2 \alpha} \hat{O}_{y}\left(\mu^{0}\right) .
\end{aligned}
$$

\subsection{A narrow tongue in parameter space}

We now define the region in the parameter space where we focus our study.

Proposition 2 Let $\varepsilon_{0}>0,0<K_{-}<K_{+}$and let

$$
\begin{aligned}
\mathcal{D}= & \left\{\left(\varepsilon_{1}, \mu\right) \mid \mu>0, \varepsilon_{1}=\frac{\mu^{2}}{4 c}-c_{1} \frac{\mu^{3}}{8 c^{3}}-G_{3}(\mu, 0)-K \mu^{4},\right. \\
& \left.0<\varepsilon_{1}<\varepsilon_{0}, K_{-} \leq K \leq K_{+}\right\} .
\end{aligned}
$$

There exists $0<\kappa_{1}<\kappa_{2}$ such that for all $\left(\varepsilon_{1}, \mu\right) \in \mathcal{D}$ and for all $y \in[-1,1]$ we have

$$
-\kappa_{2} \varepsilon_{1}^{2}<V\left(\varepsilon_{1}, \mu, y\right)<-\kappa_{1} \varepsilon_{1}^{2} .
$$

Moreover, we can choose $K_{+}$such that

$$
\sqrt{\kappa_{2}}<\frac{1}{c\left|b_{1}^{-}\right|}
$$

for $\varepsilon_{0}$ sufficiently small. 
Observe that $\mathcal{D}$ is a narrow tongue in the parameter space where the boundaries are of the form $\varepsilon_{1}=\varepsilon_{1}(\mu)$. For practical reasons, we express this tongue with boundaries of the form $\mu=\mu\left(\varepsilon_{1}\right)$. Using the same argument as in Lemma 3, writing

$$
\varepsilon_{1}=\frac{\mu^{2}}{4 c}-c_{1} \frac{\mu^{3}}{8 c^{3}}-G_{3}(\mu, 0)-K \mu^{4}
$$

amounts to writing

$$
\mu=2 \sqrt{c} \sqrt{\varepsilon_{1}}\left(1+\frac{c_{1} \sqrt{\varepsilon_{1}}}{2 c \sqrt{c}}+J\left(\varepsilon_{1}, K\right)\right)
$$

where

$$
J\left(\varepsilon_{1}, K\right)=\hat{O}_{K}\left(\varepsilon_{1} \log \left|\varepsilon_{1}\right|\right),
$$

and therefore we redefine the tongue $\mathcal{D}$ in the parameter space as follows:

$$
\begin{aligned}
\mathcal{D}= & \left\{\left(\varepsilon_{1}, \mu\right) \mid \mu=2 \sqrt{c} \sqrt{\varepsilon_{1}}\left(1+\frac{c_{1} \sqrt{\varepsilon_{1}}}{2 c \sqrt{c}}+J\left(\varepsilon_{1}, K\right)\right),\right. \\
& \left.0<\varepsilon_{1}<\varepsilon_{0}, \quad K_{-} \leq K \leq K_{+}\right\} .
\end{aligned}
$$

Proof of Proposition 2: From the above definition if $\left(\varepsilon_{1}, \mu\right) \in \mathcal{D}$, then from (19) and (20) it follows that

$$
V\left(\varepsilon_{1}, \mu, 0\right)=-K \mu^{4} \text { with } K_{-} \leq K \leq K_{+} .
$$

Furthermore, from (23) we have that

$$
V\left(\varepsilon_{1}, \mu, 0\right)=-16 K c^{2} \varepsilon_{1}^{2}+o\left(\varepsilon_{1}^{2}\right) .
$$

By the Mean Value Theorem, we have

$$
\left|V\left(\varepsilon_{1}, \mu, y\right)-V\left(\varepsilon_{1}, \mu, 0\right)\right| \leq \sup _{-1 \leq y \leq 1} \frac{\partial V}{\partial y}\left(\varepsilon_{1}, \mu, y\right) .
$$

From (19), (20) we have

$$
\frac{\partial V}{\partial y}\left(\varepsilon_{1}, \mu, y\right)=\frac{\partial G_{3}}{\partial y}(\mu, y)=O\left(\mu^{2 \alpha}\right) .
$$

Since $\alpha>2$, from (24) and (25) we have

$$
V\left(\varepsilon_{1}, \mu, y\right)=-16 K c^{2} \varepsilon_{1}^{2}+o\left(\varepsilon_{1}^{2}\right),
$$

and therefore (21) follows. Also from (24), by choosing

$$
K_{+}<1 /\left(16 c^{4}\left|b_{1}^{-}\right|^{2}\right),
$$

(22) is also satisfied for $\varepsilon_{0}$ sufficiently small.

\subsection{Fixed points}

We are in position to investigate more specific features of the dynamics. We first define an upper bound for the variable $x$. Before stating the next proposition we choose $L>1$ such that

$$
L<1+c L-2 \sqrt{c} \sqrt{L} .
$$

Observe that the existence of such $L$ is guaranteed since $c>1$.

Proposition 3 For $\varepsilon_{0}$ sufficiently small, if $\left(\varepsilon_{1}, \mu\right) \in \mathcal{D}$, then

[1] $-\kappa_{2} \varepsilon_{1}^{2} \leq \phi_{1}^{+}\left(x_{c}(y, \mu), y\right) \leq-\kappa_{1} \varepsilon_{1}^{2}$,

[2] for all $-1 \leq y \leq 1$, the function

$$
\left(0, L \varepsilon_{1}\right] \rightarrow \mathbb{R}, x \mapsto \phi_{1}^{+}(x, y)
$$

is decreasing on $\left(0, x_{c}(y, \mu)\right)$ and increasing on $\left(x_{c}(y, \mu), L \varepsilon_{1}\right)$, 
[3] there exists $\mathbf{P}_{1}=\left(P_{11}, P_{12}\right)$ such that for all $-1 \leq y \leq 1$ and for all $\left(\varepsilon_{1}, \mu\right) \in \mathcal{D}$,

$$
x_{c}(y, \mu)<\varepsilon_{1}<P_{11} \leq L \varepsilon_{1} \text { and } \phi^{+}\left(\mathbf{P}_{1}\right)=\mathbf{P}_{1} .
$$

Proof. Observe that [1] follows directly from Proposition 2. To show [2], let $y \in[-1,1]$. We know that $x_{c}(y, \mu)$ satisfies

$$
\frac{\partial \phi_{1}^{+}}{\partial x}\left(x_{c}(y, \mu), y\right)=-\frac{\mu}{2 \sqrt{x_{c}}}+c+\frac{3}{2} c_{1} x_{c}^{1 / 2}+\frac{\partial H_{1}^{+}}{\partial x}\left(x_{c}, y\right) \equiv 0
$$

Observe that

$$
\frac{\partial^{2} \phi_{1}^{+}}{\partial x^{2}}(x, y)=\frac{\mu}{4 x \sqrt{x}}+\frac{3 c_{1}}{4 \sqrt{x}}+\frac{\partial^{2} H_{1}^{+}}{\partial x^{2}}(x, y)
$$

Since $\left(\varepsilon_{1}, \mu\right) \in \mathcal{D}$, from (17) we have

$$
x_{c}=\varepsilon_{1} / c+O\left(\varepsilon_{1}^{3 / 2}\right)
$$

and with (10) we have that

$$
\frac{\partial^{2} H_{1}^{+}}{\partial x^{2}}(x, y)=\hat{O}(\log (x))+\mu x^{-3 / 2} \hat{O}(x \log |x|)+y x^{\alpha-2} \hat{O}_{y}\left(x^{0}\right) .
$$

Combining (28), (30), (23) and the fact that $0<x<L \varepsilon_{1}$ we have

$$
\frac{\partial^{2} \phi_{1}^{+}}{\partial x^{2}}(x, y)=\frac{\sqrt{\varepsilon_{1}}}{4 x \sqrt{x}}\left[2 \sqrt{c}+O\left(\sqrt{\varepsilon_{1}}\right)\right]>0 .
$$

This implies that the function $x \mapsto \phi_{1}^{+}(x, y)$ is convex on $\left[0, L \varepsilon_{1}\right]$, and we already know that it admits a critical point at $x_{c}(y, \mu)$, which implies [2]. To show [3], we introduce the function

$$
Z\left(x, y, \varepsilon_{1}, \mu\right)=\phi_{1}^{+}(x, y)-x=\varepsilon_{1}-\mu \sqrt{x}+(c-1) x+c_{1} x^{3 / 2}+H_{1}(x, y) .
$$

Observe that for $0<x \leq L \varepsilon_{1}$ and $-1 \leq y \leq 1$,

$$
\mu=2 \sqrt{c} \sqrt{\varepsilon_{1}}+O\left(\varepsilon_{1}\right) \text { and } c_{1} x^{3 / 2}+H_{1}(x, y)=O\left(\varepsilon_{1}^{3 / 2}\right) .
$$

Combining the above with (29) we have

$$
Z\left(x_{c}(y, \mu), y, \varepsilon_{1}, \mu\right)=-\varepsilon_{1} / c+O\left(\varepsilon_{1}^{3 / 2}\right)
$$

which is negative for $\varepsilon_{1}$ sufficiently small. Furthermore we have

$$
Z\left(L \varepsilon_{1}, y, \varepsilon_{1}, \mu\right)=\varepsilon_{1}\left[1-2 \sqrt{c} \sqrt{L}+(c-1) L+O\left(\sqrt{\varepsilon_{1}}\right)\right] .
$$

Since $L$ satisfies (27), there exist $\varepsilon_{3}>0$ such that for $0<\varepsilon_{1} \leq \varepsilon_{3}$ we have that

$$
Z\left(L \varepsilon_{1}, y, \varepsilon_{1}, \mu\right)>0 .
$$

By the Intermediate Value Theorem, for each $-1 \leq y \leq 1$, and for all $\left(\varepsilon_{1}, \mu\right) \in \mathcal{D}$ there exists $x_{1}=x_{1}\left(y, \varepsilon_{1}, \mu\right)$ such that

$$
x_{c}<x_{1}<L \varepsilon_{1} \text { and } Z\left(x_{1}\left(y, \varepsilon_{1}, \mu\right), y, \varepsilon_{1}, \mu\right)=0,
$$

which means that

$$
\phi_{1}^{+}\left(x_{1}\left(y, \varepsilon_{1}, \mu\right), y\right)=x_{1}\left(y, \varepsilon_{1}, \mu\right) .
$$


The choice of $x_{1}$ is, a priori, not unique. Therefore we choose $x_{1}$ to be the smallest value in $\left(x_{c}, L \varepsilon_{1}\right)$ such that (34) holds. For convenience we define

$$
\kappa_{3}=\min \left\{x_{1}\left(\varepsilon_{1}, \mu, y\right) / \varepsilon_{1}, y \in[-1,1], \quad\left(\varepsilon_{1}, \mu\right) \in \mathcal{D}\right\} .
$$

Observe that $\kappa_{3}>x_{c} / \varepsilon_{1}=1 / c+O\left(\varepsilon_{1}^{1 / 2}\right)$. Since

$$
\phi_{1}^{+}\left(x_{c}(y, \mu), y\right)<0,
$$

by the Mean Value Theorem, there exists $x_{c}<x_{2}<x_{1}$ such that

$$
\frac{\phi_{1}^{+}\left(x_{1}\left(y, \varepsilon_{1}, \mu\right), y\right)-\phi_{1}^{+}\left(x_{c}(y, \mu), y\right)}{x_{1}-x_{c}}=\frac{x_{1}-\phi_{1}^{+}\left(x_{c}(y, \mu), y\right)}{x_{1}-x_{c}}=\frac{\partial \phi_{1}^{+}}{\partial x}\left(x_{2}, y\right) .
$$

Since $\phi_{1}^{+}\left(x_{c}(y, \mu), y\right)<0$, it follows that

$$
\frac{\partial \phi_{1}^{+}}{\partial x}\left(x_{2}, y\right)>\frac{x_{1}}{x_{1}-x_{c}} .
$$

Furthermore, from (31), since $x_{2}<x_{1}$, it follows that

$$
\frac{\partial \phi_{1}^{+}}{\partial x}\left(x_{1}\left(y, \varepsilon_{1}, \mu\right), y\right)>\frac{x_{1}}{x_{1}-x_{c}}>1,
$$

which implies that $x_{1}$ is the unique value that satisfies $(34)$ on $\left(x_{c}, L \varepsilon_{1}\right)$ i.e.,

$$
Z\left(x_{1}\left(y, \varepsilon_{1}, \mu\right), y, \varepsilon_{1}, \mu\right)=0 \text { and } \frac{\partial Z}{\partial x}\left(x_{1}\left(y, \varepsilon_{1}, \mu\right), y, \varepsilon_{1}, \mu\right)>0 .
$$

Since $c>1$, from (29) we have that $x_{c}(y, \mu)<\varepsilon_{1} \leq L \varepsilon_{1}$, we therefore need to show that $\varepsilon_{1}<x_{1} \leq L \varepsilon_{1}$. Recall that for any $-1 \leq y \leq 1$

$$
\phi_{1}^{+}\left(x_{1}\left(y, \varepsilon_{1}, \mu\right), y\right)=x_{1}, \text { and } \quad \frac{\partial \phi_{1}^{+}}{\partial x}\left(x_{1}, y\right)>1,
$$

and $x_{1}$ is the unique value in $\left(x_{c}, L \varepsilon_{1}\right)$ that satisfies (34). This implies that for all $\xi \in\left(x_{c}, L \varepsilon_{1}\right)$, if

$$
\phi_{1}^{+}(\xi, y)<\xi .
$$

then $\xi \leq x_{1}$. However, since $\mu=2 \sqrt{c} \sqrt{\varepsilon_{1}}+O\left(\varepsilon_{1}\right)$ we have that

$$
\phi_{1}^{+}\left(\varepsilon_{1}, y\right)=\varepsilon_{1}-\mu \sqrt{\varepsilon_{1}}+c \varepsilon_{1}+H_{1}\left(\varepsilon_{1}, y\right)=\varepsilon_{1}\left(1-2 \sqrt{c}+c+O\left(\varepsilon_{1}^{1 / 2}\right)\right) .
$$

However, since $1<c<4$, there exists $\varepsilon_{0}>0$ such that for all $0<\varepsilon_{1} \leq \varepsilon_{0}$ the right hand side of (38) is less than $\varepsilon_{1}$. (Recall that $\left(\varepsilon_{1}, \mu\right) \in \mathcal{D}$ implies that $\varepsilon_{1}<\varepsilon_{0}$.) This shows that for all $y, \varepsilon_{1}, \mu$ we have

$$
x_{c}(y, \mu)<\varepsilon_{1}<x_{1}\left(y, \varepsilon_{1}, \mu\right) \leq L \varepsilon_{1},
$$

and as a consequence

$$
x_{c}(y, \mu) / \varepsilon_{1}<1<\kappa_{3} \leq L .
$$

Therefore

$$
\frac{1}{1-\frac{x_{c}}{L \varepsilon_{1}}}<\frac{x_{1}}{x_{1}-x_{c}}<\frac{1}{1-\frac{x_{c}}{\varepsilon_{1}}}
$$

Since $x_{c}=\varepsilon_{1} / c+O\left(\varepsilon_{1}^{3 / 2}\right)$, it follows that

$$
\frac{1}{1-\frac{x_{c}}{L \varepsilon_{1}}}=\frac{1}{1-\frac{1+O\left(\varepsilon^{1 / 2}\right)}{c L}}<\frac{x_{1}}{x_{1}-x_{c}}
$$


and therefore with (36) we have

$$
\left|1-\frac{\partial \phi_{1}^{+}}{\partial x}\left(x_{1}\left(y, \varepsilon_{1}, \mu\right), y\right)\right|^{-1} \leq c L-1+O\left(\varepsilon_{1}^{1 / 2}\right) .
$$

By the Implicit Function Theorem, we conclude that $x_{1}\left(y, \varepsilon_{1}, \mu\right)$ is a well defined $C^{1}$ function and thanks to (6), (32) and (41) we have

$$
\frac{\partial x_{1}}{\partial y}\left(y, \varepsilon_{1}, \mu\right)=\left.\frac{\partial H_{1}^{+}}{\partial y}\left(1-\frac{\partial \phi_{1}^{+}}{\partial x}\right)^{-1}\right|_{x=x_{1}\left(y, \varepsilon_{1}, \mu\right)}=O\left(x_{1}^{\alpha}\right)=O\left(\varepsilon_{1}^{\alpha}\right) .
$$

Recall that

$$
\phi_{2}^{+}(x, y)=\omega_{1}+b_{2}^{+} \sqrt{x}+H_{2}^{+}(x, y)
$$

and following (6), $H_{2}^{+}(x, y)=O(x)$ is a $C^{1}$ function with

$$
\frac{\partial H_{2}^{+}}{\partial y}(x, y)=O\left(|x|^{\alpha}\right)
$$

and therefore with (6) and (41) we have

$$
\frac{\partial}{\partial y}\left(H_{2}^{+}\left(x_{1}\left(y, \varepsilon_{1}, \mu\right), y\right)\right)=O\left(\varepsilon_{1}^{\alpha}\right) .
$$

Now define

$$
\Xi\left(y, \varepsilon_{1}, \mu\right)=\omega_{1}+b_{2}^{+} \sqrt{x_{1}\left(y, \varepsilon_{1}, \mu\right)}+H_{2}^{+}\left(x_{1}\left(y, \varepsilon_{1}, \mu\right), y\right)-y .
$$

By (29), and since $x_{c}<x_{1}$ we have

$$
\begin{aligned}
\frac{\partial x_{1}}{\partial y}\left(y, \varepsilon_{1}, \mu\right) / 2 x_{1}^{1 / 2}\left(y, \varepsilon_{1}, \mu\right) & \leq \frac{\partial x_{1}}{\partial y}\left(y, \varepsilon_{1}, \mu\right) / 2 x_{c}^{1 / 2}\left(y, \varepsilon_{1}, \mu\right) \\
& =\frac{\sqrt{c}}{2 \sqrt{\varepsilon_{1}}} \frac{\partial x_{1}}{\partial y}\left(y, \varepsilon_{1}, \mu\right)\left(1+\sqrt{\varepsilon_{1}}\right) .
\end{aligned}
$$

From (42) and (43) we have

$$
\frac{\partial \Xi}{\partial y}=-1+O\left(\varepsilon_{1}^{\alpha-1 / 2}\right) .
$$

By the Implicit Function Theorem, there exists $y=P_{12}\left(\varepsilon_{1}, \mu\right)$ such that

$$
\Xi\left(P_{12}\left(\varepsilon_{1}, \mu\right), \varepsilon_{1}, \mu\right) \equiv 0,
$$

and therefore we can define $\mathbf{P}_{1}=\left(P_{11}, P_{12}\right)$ where

$$
P_{11}\left(\varepsilon_{1}, \mu\right)=x_{1}\left(P_{12}\left(\varepsilon_{1}, \mu\right), \varepsilon_{1}, \mu\right)
$$

and together with (37) it follows that

$$
\Phi^{+}\left(\mathbf{P}_{1}\right)=\mathbf{P}_{1}
$$

Remark: Using similar arguments as for the proof of [3], one can show that there exists $\mathbf{P}_{0}=\left(P_{01}, P_{02}\right)$ such that for all for all $-1 \leq y \leq 1$ and for all $\left(\varepsilon_{1}, \mu\right) \in \mathcal{D}$,

$$
0<P_{01}<x_{c}(y, \mu) \text { and } \phi^{+}\left(\mathbf{P}_{0}\right)=\mathbf{P}_{0} .
$$

The existence of this fixed point also follows from the construction of the cuspidal horseshoe. 


\subsection{A blow-up of the parameter space}

In this section we restrict the study of the dynamics to appropriate values of the parameter $\gamma=\left(\varepsilon_{1}, \varepsilon_{2}, \mu\right)$. In order to do so we proceed as follows. We first recall that

$$
\kappa_{3}>x_{c} / \varepsilon_{1}=1 / c+O\left(\sqrt{\varepsilon_{1}}\right), \quad \sqrt{\kappa_{2}}<1 /\left(c\left|b_{1}^{-}\right|\right),
$$

and therefore for $\varepsilon_{1}$ sufficiently small, $\left|b_{1}^{-}\right| \sqrt{\kappa_{2}}<\kappa_{3}$. Let $E^{+}$and $E^{-}$be two real numbers that satisfy

$$
\left|b_{1}^{-}\right| \sqrt{\kappa_{2}}<E^{-}<E^{+}<\kappa_{3} .
$$

We define the following blow-up in the parameter space $\mathbf{B}:\left(0, \varepsilon_{0}\right) \times\left(K_{-}, K_{+}\right) \times\left(E^{-}, E^{+}\right) \rightarrow \mathbb{R}^{3}$ by

$$
\mathbf{B}\left(\varepsilon_{1}, K, E\right)=\left(\varepsilon_{1}, 2 \sqrt{c} \sqrt{\varepsilon_{1}}\left(1+T\left(\varepsilon_{1}, K\right)\right), E \varepsilon_{1}\right)
$$

where $T$ is defined from equation (23), i.e.

$$
T\left(\varepsilon_{1}, K\right)=\frac{c_{1} \sqrt{\varepsilon_{1}}}{2 c \sqrt{c}}+J\left(\varepsilon_{1}, K\right) .
$$

This means that $\left(\varepsilon_{1}, \mu\right) \in \mathcal{D}$ and $\varepsilon_{2}=E \varepsilon$ where $E \in\left(E^{-}, E^{+}\right)$if and only if

$$
\left(\varepsilon_{1}, \mu, \varepsilon_{2}\right) \in \mathbf{B}\left(\left(0, \varepsilon_{0}\right) \times\left(K_{-}, K_{+}\right) \times\left(E^{-}, E^{+}\right)\right) .
$$

Proposition 4 For all $\left(\varepsilon_{1}, K, E\right) \in\left(0, \varepsilon_{0}\right) \times\left(K_{-}, K_{+}\right) \times\left(E^{-}, E^{+}\right)$the map $\phi$ satisfies the following properties:

[1] $\left.\phi_{1}^{+}\left(\{(x, y)) \mid 0<x \leq L \varepsilon_{1}\right\}\right) \supset\left[0, L \varepsilon_{1}\right]$,

[2] $\phi^{+}\left(S^{+}\right) \cap S^{-} \subset\left\{(x, y) \mid-\kappa_{2} \varepsilon_{1}^{2}<x<0\right\}$,

[3] $\phi^{-}\left(\phi^{+}\left(S^{+}\right) \cap S^{-}\right) \subset S^{+}$.

Proof: Fix $y \in[-1,1]$ and $\left(\varepsilon_{1}, \mu\right) \in \mathcal{D}$. We know from [2] in Proposition 3 that for $x \in\left(0, L \varepsilon_{1}\right)$,

$$
\sup _{x \in\left(0, L \varepsilon_{1}\right]} \phi_{1}^{+}(x, y)=\max \left\{\varepsilon_{1}, \phi\left(L \varepsilon_{1}, y\right)\right\}
$$

Also, from (33) and (34), we know that

$$
x_{c}\left(y, \varepsilon_{1}, \mu\right)<x_{1}\left(y, \varepsilon_{1}, \mu\right)<L \varepsilon_{1}, \quad \phi_{1}\left(x_{1}\left(y, \varepsilon_{1}, \mu\right), y\right)=x_{1}\left(y, \varepsilon_{1}, \mu\right),
$$

and for all $x \geq x_{1}\left(y, \varepsilon_{1}, \mu\right)$

$$
\frac{\partial \phi_{1}^{+}}{\partial x}(x, y)>1 \text {. }
$$

This implies that $\phi_{1}^{+}\left(L \varepsilon_{1}, y\right)>L \varepsilon_{1}$ and since $L>1$, we have that

$$
\sup _{x \in\left(0, L \varepsilon_{1}\right]} \phi_{1}^{+}(x, y)=\phi\left(L \varepsilon_{1}, y\right)>L \varepsilon_{1} .
$$

Moreover, from [1] and [2] in Proposition 3, we have that for all $y \in[-1,1]$,

$$
-\kappa_{2} \varepsilon_{1}^{2}<\min _{x \in\left(0, L \varepsilon_{1}\right]} \phi_{1}^{+}(x, y)=\phi_{1}^{+}\left(x_{1}, y\right)=V\left(\varepsilon_{1}, \mu, y\right)<0 .
$$

By continuity [1] and [2] follow. As a consequence of [2], since

$$
\phi_{1}^{-}(x, y)=E \varepsilon+b_{1}^{-} \sqrt{|x|}+H_{1}^{-}(x, y), \quad H_{1}^{-}(x, y)=O(x)
$$

and $b_{1}^{-}<0$, it follows that if $-\kappa_{2} \varepsilon_{1}^{2}<x<0$,

$$
\begin{aligned}
\phi_{1}^{-}(x, y) & =E \varepsilon-\left|b_{1}^{-}\right| \sqrt{|x|}+H_{1}^{-}(x, y) \\
& >E \varepsilon-\left|b_{1}^{-}\right| \sqrt{\kappa_{2}} \varepsilon+O\left(\varepsilon^{2}\right)=\varepsilon\left(E^{-}-\left|b_{1}^{-}\right| \sqrt{\kappa_{2}}+O(\varepsilon)\right),
\end{aligned}
$$

and since

$$
E^{-}-\left|b_{1}^{-}\right| \sqrt{\kappa_{2}}>0
$$

it follows that $\phi_{1}(x, y)>0$ for $\varepsilon>0$ sufficiently small, which implies [3]. 


\subsection{The escaping band and the cuspidal horseshoe}

We now consider the region in $S^{+}$that consists of points that are mapped on $S^{-}$after the first iterate of the return map. The region contains points where the map fails to be hyperbolic. We state the following proposition.

Proposition 5 For all $\left(\varepsilon_{1}, \mu\right) \in \mathcal{D}$ there exist two curves

$$
\left\{(x, y) \mid x=x_{3}\left(y, \varepsilon_{1}, \mu\right)\right\} \text { and }\left\{(x, y) \mid x=x_{4}\left(y, \varepsilon_{1}, \mu\right)\right\}
$$

such that

$$
0<x_{3}\left(y, \varepsilon_{1}, \mu\right)<x_{4}\left(y, \varepsilon_{1}, \mu\right)<L \varepsilon_{1}
$$

and

$$
\phi_{1}^{+}\left(x_{3}\left(y, \varepsilon_{1}, \mu\right), y\right)=0=\phi_{1}^{+}\left(x_{4}\left(y, \varepsilon_{1}, \mu\right), y\right) .
$$

The proof of this proposition is a direct consequence of the Intermediate Value Theorem and the Implicit Function Theorem and follows the same steps as the proofs of [3] and [4] in Proposition 3. As a consequence of the above, both graphs $x_{3}\left(y, \varepsilon_{1}, \mu\right)$ and $x_{4}\left(y, \varepsilon_{1}, \mu\right)$ split $S^{+}$into 3 connected components:

$$
\left\{\begin{aligned}
\mathbf{H}_{0} & =\left\{(x, y) \mid 0 \leq x \leq x_{3}\left(y, \varepsilon_{1}, \mu\right)\right\} \\
\mathbf{H}_{1} & =\left\{(x, y) \mid x_{4}\left(y, \varepsilon_{1}, \mu\right) \leq x \leq L \varepsilon_{1}\right\} \\
\mathbf{N} & =\left\{(x, y) \mid x_{3}\left(y, \varepsilon_{1}, \mu\right)<x<x_{4}\left(y, \varepsilon_{1}, \mu\right)\right\} .
\end{aligned}\right.
$$

Moreover we have that

$$
\left(\phi^{+}\right)^{-1}\left(S^{+}\right) \cap S^{+} \subset \mathbf{H}_{0} \cup \mathbf{H}_{1} \text { and }\left(\phi^{+}\right)^{-1}\left(S^{-}\right) \cap S^{+}=\mathbf{N} .
$$

We now define the following sets

$$
\begin{aligned}
& \Omega_{\mathbb{N}}^{+}=\left\{(x, y) \in S^{+} \mid \phi^{n}(x, y) \in S^{+} \forall n \geq 0\right\} \text { and } \\
& \Omega_{\mathbb{Z}}^{+}=\left\{(x, y) \in S^{+} \mid \phi^{n}(x, y) \in S^{+} \forall n \in \mathbb{Z}\right\} .
\end{aligned}
$$

$\Omega_{\mathbb{N}}^{+}$is forward invariant, and $\Omega_{\mathbb{Z}}^{+}$is invariant. Since $\phi^{+}(\mathbf{N}) \subset S^{-}$, we can express both sets as follows

$$
\Omega_{\mathbb{N}}^{+}=\bigcap_{n \geq 0} \phi^{-n}\left(\mathbf{H}_{0} \cup \mathbf{H}_{1}\right) \quad \text { and } \quad \Omega_{\mathbb{Z}}^{+}=\bigcap_{n \in \mathbb{Z}} \phi^{-n}\left(\mathbf{H}_{0} \cup \mathbf{H}_{1}\right) .
$$

In order to understand the complexity of the dynamics of the full Poincare return map, we first study the dynamics restricted to $\Omega_{\mathbb{N}}^{+}$and later the dynamics on $\Omega_{\mathbb{Z}}^{+}$. We show that $\Omega_{\mathbb{N}}^{+}$inherits a hyperbolic structure, and the corresponding dynamics on $\Omega_{\mathbb{Z}}^{+}$is that of the cuspidal horseshoe. We refer here to $[6,17,38]$ for more details.

Fix $Y_{0}>2 L \varepsilon_{1}$. For convenience we define the rescaling $\mathbf{R}:[0, \sqrt{L}] \times\left[-Y_{0}-Y_{0} \omega_{1}, Y_{0}-Y_{0} \omega_{1}\right] \rightarrow \mathbb{R}^{2}$ by

$$
\mathbf{R}(u, v)=\left(R_{1}(u, v), R_{2}(u, v)\right)=\left(\varepsilon_{1} u^{2}, \frac{v}{Y_{0}}+\omega_{1}\right) .
$$

Using the fact that $\left(\epsilon_{1}, \mu\right) \in \mathcal{D}$, from (5) we have

$$
\begin{aligned}
\phi \circ \mathbf{R}(u, v)=\left(\varepsilon_{1}\left(1-2 \sqrt{c} u+c u^{2}-\frac{c_{1}}{c} \varepsilon_{1}^{1 / 2} u+c_{1} \varepsilon_{1}^{1 / 2} u^{3}+\frac{1}{\varepsilon_{1}} H_{1}^{+}\left(\varepsilon_{1} u^{2}, v / Y_{0}+\omega_{1}\right)\right),\right. \\
\left.\omega_{1}+b_{2}^{+} \sqrt{\varepsilon_{1}} u+H_{2}^{+}\left(\varepsilon_{1} u^{2}, v / Y_{0}+\omega_{1}\right)\right) .
\end{aligned}
$$

Write

$$
g(u, v)=1-2 \sqrt{c} u+c u^{2}-\frac{c_{1}}{c} \varepsilon_{1}^{1 / 2} u+c_{1} \varepsilon_{1}^{1 / 2} u^{3}+\frac{1}{\varepsilon_{1}} H_{1}^{+}\left(\varepsilon_{1} u^{2}, v / Y_{0}+\omega_{1}\right) .
$$


Observe that

$$
g(u, v)<0 \text { if and only if }(u, v) \in \mathbf{R}^{-1}(\mathbf{N})
$$

and

$$
g(u, v) \geq 0 \text { if and only if }(u, v) \in \mathbf{R}^{-1}\left(\mathbf{H}_{0} \cup \mathbf{H}_{1}\right)
$$

Denote by

$$
\mathbf{M}=\mathbf{R}^{-1}\left(\mathbf{H}_{0} \cup \mathbf{N} \cup \mathbf{H}_{1}\right), \quad \mathbf{M}^{+}=\mathbf{R}^{-1}\left(\mathbf{H}_{0} \cup \mathbf{H}_{1}\right) .
$$

We also define the following map

$$
\mathbf{F}: \mathbf{M}^{+} \rightarrow \mathbf{M},(u, v) \mapsto \mathbf{F}(u, v)=\mathbf{R}^{-1} \circ \phi \circ \mathbf{R}(u, v) .
$$

Writing $\mathbf{F}(u, v)=\left(F_{1}(u, v), F_{2}(u, v)\right)$, from (5) we have

$$
\begin{aligned}
& F_{1}(u, v)=\sqrt{g(u, v)} \\
& F_{2}(u, v)=Y_{0} b_{2}^{+} \varepsilon_{1}^{1 / 2} u+Y_{0} H_{2}^{+}\left(\varepsilon_{1} u^{2}, v / Y_{0}+\omega_{1}\right) .
\end{aligned}
$$

From (6) we have

$$
\left\{\begin{aligned}
-\frac{c_{1}}{c} \varepsilon_{1}^{1 / 2} u+c_{1} \varepsilon_{1}^{1 / 2} u^{3}+\frac{1}{\varepsilon_{1}} H_{1}^{+}\left(\varepsilon_{1} u^{2}, v / Y_{0}+\omega_{1}\right) & =\varepsilon_{1}^{1 / 2} h_{1}^{+}(u, v) \\
Y_{0} H_{2}^{+}\left(\varepsilon_{1} u^{2}, v / Y_{0}+\omega_{1}\right) & =\varepsilon_{1} h_{2}^{+}(u, v)
\end{aligned}\right.
$$

where $h_{1}^{+}$and $h_{2}^{+}$are continuous functions with bounded derivatives. Also from (6) we have

$$
\begin{aligned}
F_{1}(u, v) \frac{\partial F_{1}}{\partial v} & =O\left(\varepsilon_{1}^{\alpha-1}\right) \\
\frac{\partial F_{2}}{\partial u} & =Y_{0} b_{2}^{+} \sqrt{\varepsilon_{1}}+O\left(\varepsilon_{1}\right) \\
\frac{\partial F_{2}}{\partial v} & =O\left(\varepsilon_{1}^{\alpha}\right) .
\end{aligned}
$$
on $\mathbf{M}$

To show the hyperbolicity of the Poincaré return map restricted to $\Omega_{\mathbb{N}}^{+}$, we define the following cone field

$$
\mathcal{S}(u, v)=\left\{\left(U_{1}, U_{2}\right) \in T_{(u, v)} \mathbf{M},\left|U_{2}\right|<\left|U_{1}\right|\right\},
$$

and its complementary cone field

$$
{ }^{c} \mathcal{S}(u, v)=\left\{\left(U_{1}, U_{2}\right) \in T_{(u, v)} \mathbf{M},\left|U_{2}\right| \geq\left|U_{1}\right|\right\},
$$

and we show that these cone fields are invariant under $d \mathbf{F}$ and $d \mathbf{F}^{-1}$ respectively. We deduce the hyperbolicity from [23], see also [6, 30, 31, 37, 38]. More precisely we state the following proposition. In what follows $\|\cdot\|$ denotes the norm of the supremum, i.e.,

$$
\|\mathbf{U}\|=\left\|\left(U_{1}, U_{2}\right)\right\|=\max \left\{\left|U_{1}\right|,\left|U_{2}\right|\right\} .
$$

Observe that it is sufficient to consider the invariance of this cone field on $F\left(\mathbf{M}^{+}\right) \cap \mathbf{M}$. Equation (7) implies that $\left|p_{2} / Y_{0}+\omega_{1}\right|<1 / 2$ whenever $p \in F\left(\mathbf{M}^{+}\right) \cap \mathbf{M}$. Hence we establish the invariance of the cone field on this set.

Proposition 6 Let $\mathbf{p}=\left(p_{1}, p_{2}\right) \in \mathbf{M}^{+}$with $\left|p_{2} / Y_{0}+\omega_{1}\right|<1 / 2$. Then there exists $\lambda>1$ such that for $\varepsilon_{1}$ sufficiently small

[1] $d \mathbf{F}_{\mathbf{p}}(\mathcal{S}(\mathbf{p})) \subset \mathcal{S}(\mathbf{F}(\mathbf{p})), d \mathbf{F}_{\mathbf{F}(\mathbf{p})}^{-1}\left({ }^{c} \mathcal{S}(\mathbf{F}(\mathbf{p}))\right) \subset{ }^{c} \mathcal{S}(\mathbf{p})$,

[2] for all $\mathbf{U} \in \mathcal{S}(\mathbf{p}),\left\|d \mathbf{F}_{\mathbf{p}}(\mathbf{U})\right\| \geq \lambda\|\mathbf{U}\|$,

[3] for all $\mathbf{U} \in{ }^{c} \mathcal{S}(\mathbf{F}(\mathbf{p})),\left\|d \mathbf{F}_{\mathbf{F}(\mathbf{p})}^{-1}(\mathbf{U})\right\| \geq \lambda\|\mathbf{U}\|$. 
As a consequence of this proposition, the set $\Omega_{\mathbb{Z}}^{+}$admits a hyperbolic structure. More precisely, for each point $\mathbf{p}=\left(p_{1}, p_{2}\right) \in \Omega_{\mathbb{Z}}^{+}$there exists $x_{0}>0$ and a leaf

$$
\mathcal{F}_{x_{0}}=\left\{(x, y) \in S^{+} \mid x=\mathcal{F}_{x_{0}}(y), \mathcal{F}_{x_{0}}(0)=x_{0}, \quad-1 \leq y \leq 1\right\}
$$

such that

$$
\mathbf{p} \in \mathcal{F}_{x_{0}} \text { and } \phi\left(\mathcal{F}_{x_{0}}\right) \subset \mathcal{F}_{\phi(\mathbf{p})_{1}}
$$

see $[23,31,30]$ for more details. See also $[6,17,38,35]$ for other references. In particular there exists $d>0$ such that the leaf

$$
\mathcal{F}_{d}=\left\{(x, y) \in S^{+} \mid x=\mathcal{F}_{d}(y), \quad-1 / 2 \leq y \leq 1 / 2\right\}
$$

contains the fixed point $\mathbf{P}_{1}=\left(P_{11}, P_{12}\right)$ and is invariant, i.e.

$$
\mathcal{F}_{d}\left(P_{12}\right)=P_{11} \text { and } \mathbf{F}\left(\mathcal{F}_{d}\right) \subset \mathcal{F}_{d}
$$

We complete this lamination by constructing an invariant foliation

$$
\mathcal{F}=\left\{\mathcal{F}_{x_{0}} \mid x_{0} \in\left[0, L \varepsilon_{1}\right]\right\}
$$

on $\mathbf{M}$ as follows.

First, if $\mathbf{p} \in \Omega_{\mathbb{Z}}^{+}$we define the foliation as being the one described above using Proposition 6 . Next, we extend $\mathcal{F}$ to $\mathbf{N}$ by choosing an arbitrary smooth foliation on $\mathbf{N}$. Finally, if $\mathbf{p} \notin \Omega_{\mathbb{Z}}^{+}$, then there exists a smallest integer $n \geq 0$ such that $\phi^{n}(\mathbf{p}) \in \mathbf{N}$. Let $\mathcal{F}_{x_{n}}$ be the leaf of $\mathcal{F}$ passing through $\phi^{n}(\mathbf{p})$. We define the leaf passing through $\mathbf{p}$ as the connected component of $\phi^{-n}\left(\mathcal{F}_{x_{n}}\right)$ containing $\mathbf{p}$. This defines the construction of the foliation $\mathcal{F}$ on $S^{+}$. See $[30,38]$ for more details.

We further extend the construction of the foliation to $S^{-}$using property [3] of Proposition 4 . A point $\mathbf{p}$ in $S^{-}$is mapped to $\phi(\mathbf{p}) \in S^{+}$. Let $\mathcal{F}_{x_{0}}$ be the leaf of $\mathcal{F}$ passing through $\phi(\mathbf{p})$. We define the leaf passing through p as

$$
\phi^{-1}\left(\mathcal{F}_{x_{0}} \cap \phi\left(S^{-}\right)\right) .
$$

As a consequence, this defines a foliation on $S^{+} \cup S^{-}$that is $C^{1}$, i.e, there exists a $C^{1}$ diffeomorphism

$$
\Phi:[-1 / 2,0) \cup(0,1] \times[-1,1] \rightarrow S^{+} \cup S^{-}
$$

such that each leaf of the foliation $\mathcal{F}$ defined above is the image under $\Phi$ of lines of the form $\{x=$ const $\}$. In this new system of coordinates the Poincaré return map satisfies properties (i)-(vii) in Section 1.1.

Remark 1 This completes the proof of Theorem 1 assuming Proposition 6. The proof of Proposition 6 relies on the following lemma.

Lemma 4 Let $I$ be an interval containing 0 and $s: I \rightarrow \mathbb{R}$ be a $C^{2}$ function that satisfies

[i] $s^{\prime}(p)=0$ and $s(p)<0$ for some $p \in I$,

[ii] there exists $\eta>1$ such that for all $\xi_{1}, \xi_{2} \in I,\left(s^{\prime \prime}\left(\xi_{2}\right)\right)^{2} / 2 s^{\prime \prime}\left(\xi_{1}\right)>\eta$.

Then for all $z$ such that $s(z)>0$

$$
\left|\frac{d}{d z} \sqrt{s(z)}\right|>\sqrt{\eta} .
$$

Proof. Applying the Taylor-Lagrange Remainder Theorem at the point $p$, we have

$$
\begin{aligned}
& s(z)=s(p)+s^{\prime}(p)(z-p)+\frac{s^{\prime \prime}\left(\xi_{1}\right)}{2}(z-p)^{2}=s(p)+\frac{s^{\prime \prime}\left(\xi_{1}\right)}{2}(z-p)^{2} \\
& \text { and } \\
& s^{\prime}(z)=s^{\prime}(p)+s^{\prime \prime}\left(\xi_{2}\right)(z-p)=s^{\prime \prime}\left(\xi_{2}\right)(z-p)
\end{aligned}
$$

for some $\xi_{1}, \xi_{2} \in[\min \{p, z\}, \max \{p, z\}]$. Notice that

$$
\frac{d}{d z} \sqrt{s(z)}=\frac{s^{\prime}(z)}{2 \sqrt{s(z)}}=\frac{s^{\prime \prime}\left(\xi_{2}\right)(z-p)}{2\left(s(p)+\frac{s^{\prime \prime}\left(\xi_{1}\right)}{2}(z-p)^{2}\right)^{1 / 2}} .
$$


After squaring the previous expression, we get

$$
\left(\frac{d}{d z} \sqrt{s(z)}\right)^{2}=\frac{\left(s^{\prime \prime}\left(\xi_{2}\right)\right)^{2}(z-p)^{2}}{4\left(s(p)+\frac{s^{\prime \prime}\left(\xi_{1}\right)}{2}(z-p)^{2}\right)},
$$

and since $s(p)<0$, we have that

$$
\frac{\left(s^{\prime \prime}\left(\xi_{2}\right)\right)^{2}(z-p)^{2}}{4\left(s(p)+\frac{s^{\prime \prime}\left(\xi_{1}\right)}{2}(z-p)^{2}\right)}>\frac{\left(s^{\prime \prime}\left(\xi_{2}\right)\right)^{2}(z-p)^{2}}{\left(4 \frac{s^{\prime \prime}\left(\xi_{1}\right)}{2}(z-p)^{2}\right)}
$$

Therefore from [ii]

$$
\left(\frac{d}{d z} \sqrt{s(z)}\right)^{2}>\frac{\left(s^{\prime \prime}\left(\xi_{2}\right)\right)^{2}}{2 s^{\prime \prime}\left(\xi_{1}\right)}>\eta
$$

which completes the proof of the lemma.

Proof of Proposition 6. Let $\mathbf{p}=\left(p_{1}, p_{2}\right) \in \mathbf{M}^{+}$and $\left(U_{1}, U_{2}\right) \in \mathcal{S}(\mathbf{p})$ i.e, $\left|U_{2}\right|<\left|U_{1}\right|$, and write

$$
d \mathbf{F}_{\mathbf{p}}\left(U_{1}, U_{2}\right)=\left(\begin{array}{cc}
\frac{\partial F_{1}}{\partial u}(\mathbf{p}) & \frac{\partial F_{1}}{\partial v}(\mathbf{p}) \\
\frac{\partial F_{2}}{\partial u}(\mathbf{p}) & \frac{\partial F_{2}}{\partial v}(\mathbf{p})
\end{array}\right) \cdot\left(\begin{array}{c}
U_{1} \\
\\
U_{2}
\end{array}\right) .
$$

We want to show that

$$
\left|\frac{\partial F_{1}}{\partial u}(\mathbf{p}) U_{1}+\frac{\partial F_{1}}{\partial v}(\mathbf{p}) U_{2}\right|>\left|\frac{\partial F_{2}}{\partial u}(\mathbf{p}) U_{1}+\frac{\partial F_{2}}{\partial v}(\mathbf{p}) U_{2}\right|,
$$

or equivalently, after division by $\left|U_{1}\right|$,

$$
\left|\frac{\partial F_{1}}{\partial u}(\mathbf{p})+\frac{\partial F_{1}}{\partial v}(\mathbf{p}) U\right|>\left|\frac{\partial F_{2}}{\partial u}(\mathbf{p})+\frac{\partial F_{2}}{\partial v}(\mathbf{p}) U\right|
$$

where $U=U_{2} / U_{1}$ with $|U|<1$. From equations (52) and (53) we have

$$
\frac{\partial F_{2}}{\partial u}=O\left(\sqrt{\varepsilon_{1}}\right), \quad \frac{\partial F_{2}}{\partial v}=O\left(\varepsilon_{1}^{\alpha}\right)
$$

so that it is sufficient to show that

$$
\left|\frac{\partial F_{1}}{\partial u}(\mathbf{p})+\frac{\partial F_{1}}{\partial v}(\mathbf{p}) U\right|>\sqrt{\eta}
$$

for some $\eta>1$.

Consider therefore the line $\ell$ parametrized by $z$ and directed by the vector $(1, U)$ passing through the point $\mathbf{p}=\left(p_{1}, p_{2}\right)$, i.e.

$$
\ell(z)=\left(p_{1}+z, p_{2}+z U\right)
$$

It follows that

$$
\frac{\partial F_{1}}{\partial u}(\ell(z))+\frac{\partial F_{1}}{\partial v}(\ell(z)) U=D_{(1, U)} F_{1}(\ell(z))=\frac{\partial}{\partial z} F_{1}(\ell(z))
$$

i.e. the directional derivative of $F_{1}$ in the direction $(1, U)$ at the point $\ell(z)$. From equations $(51)$ and $(52)$ we write

$$
g(z)=1-2 \sqrt{c}\left(p_{1}+z\right)+c\left(p_{1}+z\right)^{2}+\varepsilon_{1}^{1 / 2} h_{1}^{+}\left(p_{1}+z, p_{2}+U z\right),
$$


and $g(z)=\left(F_{1}(\ell(z))\right)^{2}$ whenever $g(z)>0$. Since $\left|p_{2} / Y_{0}+\omega_{1}\right|<1 / 2$ and $L \varepsilon_{1}<Y_{0} / 2$, there exists $z_{0} \in \mathbb{R}$ such that

$$
\begin{aligned}
x_{3}\left(R_{2}\left(p_{1}+z_{0}, v+z_{0} U\right), \varepsilon_{1}, \mu\right) & <R_{1}\left(p_{1}+z_{0}, p_{2}+z_{0} U\right)=\varepsilon_{1}\left(p_{1}+z_{0}\right)^{2} \\
& <x_{4}\left(R_{2}\left(p_{1}+z_{0}, p_{2}+z_{0} U\right), \varepsilon_{1}, \mu\right)
\end{aligned}
$$

i.e. $\ell\left(z_{0}\right)=\left(p_{1}+z_{0}, p_{2}+z_{0} U\right) \in \mathbf{R}^{-1}(\mathbf{N})$, and therefore $g\left(z_{0}\right)<0$. Also observe that

$$
g^{\prime \prime}(z)=2 c+O\left(\varepsilon_{1}^{1 / 2}\right)
$$

which implies that $\left(g^{\prime \prime}(z)\right)^{2}=4 c^{2}+O\left(\varepsilon_{1}^{1 / 2}\right)$, and thus for sufficiently small $\varepsilon_{1}$ and for all $\xi_{1}, \xi_{2}$ we have

$$
\left(g^{\prime \prime}\left(\xi_{2}\right)\right)^{2} / 2 g^{\prime \prime}\left(\xi_{1}\right)=c+O\left(\varepsilon_{1}^{1 / 2}\right)>(1+c) / 2 .
$$

This means that $g$ satisfies the assumption of Lemma 4, and we conclude that

$$
(\sqrt{g(z)})^{\prime}=\frac{\partial}{\partial z} F_{1}(\ell(z))>(1+c) / 2
$$

whenever $g(z)>0$. In particular, $\ell(0)=\mathbf{p} \in \mathbf{M}^{+}$so that $g(0)>0$, which completes the proof of statement [1] in Proposition 6.

Also from (56) it follows that

$$
\left|\frac{\partial F_{1}}{\partial u}(\mathbf{p}) U_{1}+\frac{\partial F_{1}}{\partial v}(\mathbf{p}) U_{2}\right|>\eta\left|U_{1}\right|=\eta\|\mathbf{U}\|,
$$

and therefore statement [2] follows. Observe that from (57) and the triangle inequality we have

$$
\left|\frac{\partial F_{1}}{\partial u}(\mathbf{p})\right|>\eta-O\left(\varepsilon_{1}\right)
$$

and for $\varepsilon_{1}$ sufficiently small, we have that

$$
\left|\frac{\partial F_{1}}{\partial u}(\mathbf{p})\right|>1+\eta / 2 .
$$

We consider now a vector $\mathbf{U}=\left(U_{1}, U_{2}\right) \in^{c} \mathcal{S}(\mathbf{F}(\mathbf{p}))$, i.e. $\left|U_{2}\right| \geq\left|U_{1}\right|$. We want to show that

$$
\left\|d \mathbf{F}_{\mathbf{F}(\mathbf{p})}^{-1}(\mathbf{U})\right\| \geq \lambda\|\mathbf{U}\|
$$

for some $\lambda>1$. By the Inverse Function Theorem, we have that

$$
d \mathbf{F}_{\mathbf{F}(\mathbf{p})}^{-1}=\frac{1}{\Delta}\left(\begin{array}{cc}
\frac{\partial F_{2}}{\partial v}(\mathbf{p}) & -\frac{\partial F_{1}}{\partial v}(\mathbf{p}) \\
-\frac{\partial F_{2}}{\partial u}(\mathbf{p}) & \frac{\partial F_{1}}{\partial u}(\mathbf{p})
\end{array}\right)
$$

where

$$
\Delta=\frac{\partial F_{1}}{\partial u}(\mathbf{p}) \frac{\partial F_{2}}{\partial v}(\mathbf{p})-\frac{\partial F_{1}}{\partial v}(\mathbf{p}) \frac{\partial F_{2}}{\partial u}(\mathbf{p}) .
$$

From (58), (52), and (53) we have

$$
|\Delta|=\sqrt{\varepsilon_{1}} \frac{\partial F_{1}}{\partial u} \mathbf{B}(u, v)
$$

where $\mathbf{B}$ is a bounded function on $\mathbf{H}_{0} \cup \mathbf{H}_{\mathbf{1}}$, say

$$
|\mathbf{B}(u, v)| \leq \mathbf{K} .
$$

We need to show that if $\left|U_{1}\right| \leq\left|U_{2}\right|$, then

$$
\frac{1}{|\Delta|}\left|-\frac{\partial F_{2}}{\partial u}(\mathbf{p}) U_{1}+\frac{\partial F_{1}}{\partial u}(\mathbf{p}) U_{2}\right|>\lambda\left|U_{2}\right|
$$


for some $\lambda>1$. Denote by $W=U_{1} / U_{2}$. After division by $\left|U_{2}\right|$, this amounts to showing

$$
\frac{1}{|\Delta|}\left|-\frac{\partial F_{2}}{\partial u}(\mathbf{p}) W+\frac{\partial F_{1}}{\partial u}(\mathbf{p})\right|>\lambda .
$$

With (61), (58) and (53) it follows that

$$
\begin{aligned}
\frac{1}{|\Delta|}\left|-\frac{\partial F_{2}}{\partial u}(\mathbf{p}) W+\frac{\partial F_{1}}{\partial u}(\mathbf{p})\right| & =\frac{1}{\mathbf{B}(u, v) \sqrt{\varepsilon_{1}}}\left|-\left(\frac{\partial F_{2}}{\partial u}(\mathbf{p}) / \frac{\partial F_{1}}{\partial u}(\mathbf{p})\right) W+1\right| \\
& \geq \frac{1}{\mathbf{K} \sqrt{\varepsilon_{1}}}|1-| \frac{-b_{+} \sqrt{\varepsilon_{1}}+O\left(\varepsilon_{1}\right)}{(1+\eta / 2)} W||
\end{aligned}
$$

and therefore for $\varepsilon_{1}>0$ sufficiently small

$$
\frac{1}{|\Delta|}\left|-\frac{\partial F_{2}}{\partial u}(\mathbf{p}) W+\frac{\partial F_{1}}{\partial u}(\mathbf{p})\right| \geq \frac{1}{2 \mathbf{K} \sqrt{\varepsilon_{1}}} \geq 2,
$$

which completes the proof of the proposition.

\section{Computational results}

In this section we present a computational analysis of a map, see equation (71), obtained from the Poincaré map in (5) after scaling by $\varepsilon_{1}$ and dropping higher-order terms. The choices of specific parameters in (71) are made for computational convenience. First we briefly discuss combinatorial outer approximations and the use of Conley index theory as tools to analyze dynamical systems from a computational point of view. A combinatorial outer approximation of a function $f: X \rightarrow X$ is a finite representation of the dynamical system generated by iterating $f$ that is compatible with tools from Conley index theory in two ways. First, it incorporates round-off errors that occur in its construction so that the derived results are rigorous. Second, it is a combinatorial object to which tools from computational topology and graph theory can be applied. We keep the discussion of outer approximations and Conley index theory brief and refer the reader to $[20,9,8,7,1,2]$ and references therein for more details.

\subsection{Outer approximations}

We begin the construction of an outer approximation by discretizing the phase space $X$ into a finite grid of rectangular boxes $\mathcal{X}$ on which we compute image bounds. For a subset of boxes $\mathcal{B} \subset \mathcal{X}$ define the topological realization of $\mathcal{B}$ as $|\mathcal{B}|:=\cup_{B \in \mathcal{B}} B \subset \mathbb{R}^{d}$.

Constructing an outer approximation of $f$ on $\mathcal{X}$ involves computing an outer bound on the image $f(B)$ for each $B \in \mathcal{X}$. In our approach, we use interval arithmetic calculations with outward rounding to compute rigorously a rectangular outer bound on $f(B)$, which we then intersect with the grid $\mathcal{X}$. The corresponding outer approximation is a multivalued map $\mathcal{F}: \mathcal{X} \rightrightarrows \mathcal{X}$ where for $B \in \mathcal{X}$

$$
f(B) \subset \operatorname{int}|\mathcal{F}(B)| \text {. }
$$

The outer approximation $\mathcal{F}$ can be represented by a directed graph for computational purposes. In the directed graph representation, the vertex set is the set of boxes in the grid, and there is a directed edge from vertex $B$ to vertex $B^{\prime}$ if and only if $B^{\prime} \in \mathcal{F}(B)$.

\subsection{Computational Conley index theory}

Computational Conley index theory is one tool that can be applied to outer approximations in order to draw rigorous conclusions about the dynamics of the original system. We begin with an extension of dynamical systems terminology to outer approximations followed by the definitions of isolating neighborhood and index pair, which are the building blocks of Conley index theory.

Definition 1 A combinatorial trajectory of $\mathcal{F}$ through $B \in \mathcal{X}$ is a bi-infinite sequence $\gamma_{B}=\left(\ldots, B_{-1}, B_{0}, B_{1}, \ldots\right)$ with $B_{0}=B$ and $B_{n+1} \in \mathcal{F}\left(B_{n}\right)$ for all $n \in \mathbb{Z}$. 
Recall that a trajectory of $f$ through $x \in X$ is a sequence

$$
\gamma_{x}:=\left(\ldots, x_{-1}, x_{0}, x_{1}, \ldots\right)
$$

such that $x_{0}=x$ and $x_{n+1}=f\left(x_{n}\right)$ for all $n \in \mathbb{Z}$. Note that given an outer approximation $\mathcal{F}$ of $f$ and a trajectory $\gamma_{x}:=\left(\ldots, x_{-1}, x_{0}, x_{1}, \ldots\right)$ of $f, \gamma_{B_{0}}=\left(\ldots, B_{-1}, B_{0}, B_{1}, \ldots\right)$ where $x_{i} \in B_{i}$ is a trajectory for $\mathcal{F}$. We now define the invariant set relative to $N \subset X$ as

$$
\operatorname{Inv}(N, f):=\left\{x \in N \mid \text { there exists a trajectory } \gamma_{x} \text { of } f \text { with } \gamma_{x} \subset N\right\} .
$$

Definition 2 The combinatorial invariant set relative to $\mathcal{N} \subset \mathcal{X}$ for a multivalued map $\mathcal{F}$ is

$$
\operatorname{Inv}(\mathcal{N}, \mathcal{F}):=\left\{B \in \mathcal{X} \mid \text { there exists a trajectory } \gamma_{B} \text { of } \mathcal{F} \text { with } \gamma_{B} \subset \mathcal{N}\right\}
$$

Conley index theory pertains to invariant sets in isolating neighborhoods.

Definition 3 Let $X$ be a locally compact metric space. A compact set $N \subset X$ is an isolating neighborhood for $g: X \rightarrow X$ if

$$
\operatorname{Inv}(N, g) \subset \operatorname{int}(N)
$$

where $\operatorname{int}(N)$ denotes the interior of $N$. A set $S$ is an isolated invariant set if $S=\operatorname{Inv}(N, f)$ for $\operatorname{some}$ isolating neighborhood $N$.

While there are different sufficient conditions for isolation in the setting of outer approximations, we chose the following for this work. The set $o(\mathcal{B}):=\{B \in \mathcal{X}|B \cap| \mathcal{B} \mid \neq \emptyset\}$, sometimes referred to as a one box neighborhood of $\mathcal{B}$ in $\mathcal{X}$, provides the smallest representable neighborhood $|o(\mathcal{B})|$ of $|\mathcal{B}|$ in the grid $\mathcal{X}$. If

$$
o(\operatorname{Inv}(\mathcal{N}, \mathcal{F})) \subset \mathcal{N}
$$

then $\mathcal{N} \subset \mathcal{X}$ is a combinatorial isolating neighborhood under $\mathcal{F}$.

By construction, the topological realization $|\mathcal{N}|$ of a combinatorial isolating neighborhood $\mathcal{N}$ under $\mathcal{F}$ is an isolating neighborhood for $f$. This definition is stronger than what is actually required to guarantee isolation on the topological level. It is, however, the definition that we use in this work and is computable using the following algorithm.

Let $\mathcal{S} \subset \mathcal{X}$. Set $\mathcal{N}=\mathcal{S}$ and let $o(\mathcal{N})$ be the combinatorial neighborhood of $\mathcal{N}$ in $\mathcal{X}$. If $\operatorname{Inv}(o(\mathcal{N}), \mathcal{F})=\mathcal{N}$, then $\mathcal{N}$ is isolated under $\mathcal{F}$. If not, set $\mathcal{N}:=\operatorname{Inv}(o(\mathcal{N}), \mathcal{F})$ and repeat the above procedure. In this way, we grow the set $\mathcal{N}$ until either the isolation condition is met, or the set grows to intersect the boundary of $\mathcal{X}$ in which case the algorithm fails to locate an isolating neighborhood in $\mathcal{X}$. However, if the set on which the algorithm is applied is an attractor, then a neighborhood that intersects the boundary is permissible. This procedure for growing a combinatorial isolating neighborhood is outlined in more detail in [9] and [8].

Once we have an isolating neighborhood for $f$, our next goal is to compute a corresponding index pair. The following definition of a combinatorial index pair emphasizes use of an outer approximation to compute structures for $f$.

Definition 4 (Robbin and Salamon, [33]) Let $P=\left(P_{1}, P_{0}\right)$ be a pair of compact sets with $P_{0} \subset P_{1} \subset X$. $P$ is an index pair provided that $\operatorname{cl}\left(P_{1} \backslash P_{0}\right)$ is an isolating neighborhood and the induced map, $f_{P}:\left(P_{1} / P_{0},\left[P_{0}\right]\right) \rightarrow$ $\left(P_{1} / P_{0},\left[P_{0}\right]\right)$,

$$
f_{P}(x):= \begin{cases}f(x) & \text { if } x, f(x) \in P_{1} \backslash P_{0} \\ {\left[P_{0}\right]} & \text { otherwise }\end{cases}
$$

is continuous. Finally, a pair $\mathcal{P}=\left(\mathcal{P}_{1}, \mathcal{P}_{0}\right)$ of cubical sets is a combinatorial index pair for an outer approximation $\mathcal{F}$ if the corresponding topological realization $P=\left(P_{1}, P_{0}\right)$, where $P_{i}:=\left|\mathcal{P}_{i}\right|$, is an index pair for $f$.

An algorithm is given in [8] that can be used to compute a combinatorial index pair corresponding to a combinatorial isolating neighborhood. In essence, the algorithm identifies the portions of the boundary of the combinatorial isolating neighborhood that act as an exit set, meaning that trajectories that leave the neighborhood must (topologically) pass through this set. The second element of the pair, $\mathcal{P}_{0}$, records the exit set.

Given an outer approximation, we now have algorithms to compute isolating neighborhoods $|\mathcal{N}|$ and corresponding index pairs $P:=\left(\left|\mathcal{P}_{1}\right|,\left|\mathcal{P}_{0}\right|\right)$ for $f$, where $|\mathcal{N}|=\left|\mathcal{P}_{1} \backslash \mathcal{P}_{0}\right|$. What remains is the computation of the Conley index for the associated isolated invariant set, $S:=\operatorname{Inv}(|\mathcal{N}|, f)$. 
Definition 5 Let $P=\left(P_{1}, P_{0}\right)$ be an index pair for the isolated invariant set $S=\operatorname{Inv}\left(\operatorname{cl}\left(P_{1} \backslash P_{0}\right), f\right)$ and let $f_{P *}: H_{*}\left(P_{1}, P_{0}\right) \rightarrow H_{*}\left(P_{1}, P_{0}\right)$ be the maps induced on the relative homology groups $H_{*}\left(P_{1}, P_{0}\right)$ from the map $f_{P}$. The Conley index of $S$ is the shift equivalence class of $f_{P *}$

$$
\operatorname{Con}(S, f):=\left[f_{P_{*}}\right]_{s} .
$$

For a definition of shift equivalence, see [12].

The Conley index for the isolated invariant set $S$ given in Definition 5 is well-defined, namely, every isolated invariant set has an index pair, and the corresponding shift equivalence class remains invariant under different choices for this index pair, see e.g. [22].

What remains in the computation of the index is to compute the maps $f_{P_{*}}: H_{*}\left(\left|\mathcal{P}_{1}\right|,\left|\mathcal{P}_{0}\right|\right) \rightarrow H_{*}\left(\left|\mathcal{P}_{1}\right|,\left|\mathcal{P}_{0}\right|\right)$. If the multivalued map $\mathcal{F}$ is acyclic on $\mathcal{P}_{1}$, that is images of individual boxes in $\mathcal{P}_{1}$ have the topology of a point, then once again the combinatorial multivalued map provides the appropriate computational framework for computing these induced maps on homology as described in [18], and we use the software program homcubes in [32] to check acyclicity and compute $f_{P *}$. This step is also outlined in [8].

So far we have a way to pass from continuous maps to induced maps on relative homology. Our overall goal, however, is to describe the dynamics of our original map. There are a number of tools that one can use to interpret Conley indices. The most basic is the Ważewski property of the index.

Theorem 2 If $\operatorname{Con}(S, f) \neq[0]_{s}$, then $S \neq \emptyset$.

In addition, we may use the Lefschetz number to draw more detailed conclusions about the dynamics.

Theorem 3 Let $P=\left(P_{1}, P_{0}\right)$ be an index pair for isolated invariant set $S$. If the Lefschetz number

$$
L(S, f):=\sum_{k}(-1)^{k} \operatorname{tr}\left(f_{P k}\right)
$$

is nonzero, then $S$ contains a fixed point.

The Lefschetz number of the isolated invariant set $S$ is well-defined, since the Conley index of $S$ is well-defined and the trace is invariant under shift equivalence.

By attaching symbols to each connected component of the isolating neighborhood and computing Conley index information for maps between labeled regions, one may use an extension of Theorem 3 to study symbolic dynamics, as described in the following theorem and corollary.

Theorem 4 Let $N \subset X$ be the union of disjoint, compact sets $N_{1}, \ldots, N_{m}$ and let $S:=\operatorname{Inv}(N, f)$ be the isolated invariant set relative to $N$. Let $S^{\prime}=\operatorname{Inv}\left(N_{1},\left.\left.f\right|_{N_{n}} \circ \cdots \circ f\right|_{N_{1}}\right) \subset S$ where $\left.f\right|_{N_{i}}$ denotes the restriction of the map $f$ to the region $N_{i}$. If $\operatorname{Con}\left(S^{\prime},\left.\left.f\right|_{N_{n}} \circ \cdots \circ f\right|_{N_{1}}\right) \neq[0]_{s}$, then $S^{\prime} \neq \emptyset$. More specifically, there exists a point in $S$ whose trajectory under $f$ travels through the regions $N_{1}, \ldots, N_{n}$ in the prescribed order.

Corollary 1 If $L\left(S^{\prime},\left.f\right|_{N_{n}} \circ \cdots \circ f_{N_{1}}\right) \neq 0$, then $\left.f\right|_{N_{n}} \circ \cdots \circ f_{N_{1}}$ contains a fixed point in $S^{\prime}$ that corresponds to a periodic point of period $n$ in $S$ that under $f$ travels through the regions $N_{1}, \ldots, N_{n}$ in order.

Using the above corollary, Day, et al. [8] develop techniques to compute and validate a semiconjugacy from $f$ to a symbolic dynamical system. From this one may also obtain rigorous lower bounds on topological entropy, one measure of chaos. This approach is described in more detail in [8] and is applied to the outer approximation of the truncated Poincaré map of the re-injected cuspidal horseshoe. Some earlier algorithms and results using these ideas can be found in [40, 41].

\subsection{Computational results}

In this section we consider the two-dimensional map

$$
\begin{aligned}
& \varphi^{+}(x, y)=\left[\begin{array}{c}
1-M \sqrt{x}+c x-6 \epsilon^{\alpha-1} x^{\alpha} y \\
0.3-3 \sqrt{\epsilon x}-2 \epsilon^{\alpha} x^{\alpha} y
\end{array}\right] \\
& \varphi^{-}(x, y)=\left[\begin{array}{c}
0.65+0.4 \sqrt{\frac{|x|}{\epsilon}}+0.4 x+240|x|^{\alpha} y \\
-0.225+(6 \sqrt{\epsilon}+0.25) \sqrt{|x|}-2 \epsilon^{\alpha}|x|^{\alpha} y
\end{array}\right]
\end{aligned}
$$



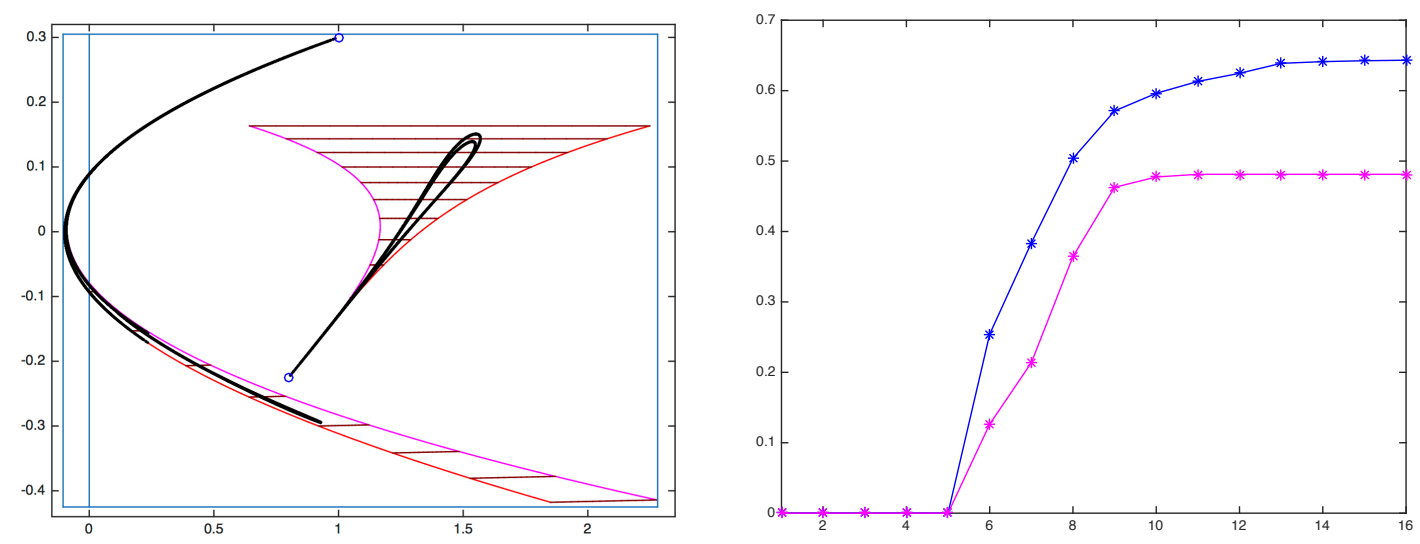

Figure 5: (a) Images $\varphi^{+}\left(S^{+}\right)$and $\varphi^{-}\left(S^{-}\right)$. The cusps (blue circles) at $(1,0.3)$ and $(0.65,-0.225)$ are the right-hand limit of $\varphi^{+}$as $x \rightarrow 0^{+}$and left-hand limit of $\varphi^{-}$as $x \rightarrow 0^{-}$respectively. The black points are 30,000 iterates of a single orbit. (b) Rigorous lower bounds on topological entropy for the full system (blue) and for the cuspidal horseshoe without re-injection (magenta). The horizontal axis is the upper bound on the period of the boxes used to seed the index pair computation, see text for a full explanation.

where $c=2.75, M=3.47098748700613, \epsilon=0.025$, and $\alpha=2.1$. The domain is the union of rectangles $S^{+}=[-0.105,0) \times[-0.425,0.305]$ and $S^{-}=(0,2.28] \times[-0.425,0.305]$ with $\varphi^{+}$acting on $S^{+}$and $\varphi^{-}$acting on $S^{-}$. The image $\varphi^{+}\left(S^{+}\right) \cup \varphi^{-}\left(S^{-}\right)$is shown in Figure $5($ a). The cusps, shown as blue circles, at $(1,0.3)$ and $(0.65,-0.225)$ are the right-hand limit of $\varphi^{+}$as $x \rightarrow 0^{+}$and left-hand limit of $\varphi^{-}$as $x \rightarrow 0^{-}$respectively. We also plot 30,000 points of a single orbit in black.

For more efficient computation, we restrict to the rectangular domain $S=[-0.1009375,1.4190625] \times$ $[-0.266,0.30]$, which contains the invariant set, as indicated in Figure 5 . After subdividing the domain 29 times, so that the size of each box is approximately $4.6 \times 10^{-5}$ by $3.5 \times 10^{-5}$, we compute an outer approximation $\mathcal{F}$ of $\varphi^{ \pm}$on $\left(S \cap S^{+}\right) \cup\left(S \cap S^{-}\right)$. Note that the vertical line $\{x=0\}$ is a subdivision line after 9 subdivisions in the horizontal direction, so each box belongs to either $S^{+}$or $S^{-}$. The image of a box whose boundary intersects the line $\{x=0\}$ is closed using the right-hand limit of $\varphi^{+}$as $x \rightarrow 0^{+}$, which is the cusp $(1,0.3)$, and the left-hand limit of $\varphi^{-}$as $x \rightarrow 0^{-}$, which is the cusp $(0.65,-0.225)$.

For $p=1, \ldots, 16$ we extract the set of boxes whose first return time in the graph of $\mathcal{F}$ is at most $p$, that is, each box in this set lies on a cycle of $\mathcal{F}$ with period at most $p$. Then we remove boxes that are within a horizontal distance of four box diameters of the line $\{x=0\}$ and grow an isolating neighborhood beginning with the recurrent part of the remaining boxes. In each case isolation is attained without intersecting the line $\{x=0\}$ or the boundary of the domain. We then compute an index pair and a semiconjugacy to a symbolic dynamical system. From each of these symbolic systems we compute the topological entropy, which provide rigorous lower bounds on the topological entropy of the original map $\varphi^{ \pm}$in these isolated invariant sets. The results are plotted in blue in Figure 5(b). A sample index pair, obtained by starting from boxes with first return time at most 11, is shown in Figure 6(a).

Next we compare the above results for the full system to those for the cuspidal horseshoe without reinjection by analyzing the map $\varphi^{+}$on the domain $[0,1.05] \times[-0.2,0.305]$. As before, for $p=1, \ldots, 16$ we extract the set of boxes whose first return time in the graph of $\mathcal{F}$ is at most $p$. Then we remove boxes that are within a horizontal distance of four box diameters of the line $\{x=0\}$ and grow an isolating neighborhood beginning with the recurrent part of the remaining boxes. In each case isolation is attained without intersecting the line $\{x=0\}$ or the boundary of the domain, and we compute an index pair. The resulting lower bounds on the topological entropy in this case are plotted in magenta in the Figure 5(b).

Using 29 subdivisions of the domain, corresponding to an approximate box size $3.2 \times 10^{-5}$ by $3.1 \times 10^{-5}$, every recurrent box, i.e. a box lying on a cycle of $\mathcal{F}$, has first return time at most 15 . Figure $6(\mathrm{~b})$ shows the index pair computed from the entire recurrent set. As shown, the index pair can be amalgamated to 3 sets of boxes labeled A, B, C. The dynamics of $f$ is then semiconjugate to a symbolic dynamical system on three 

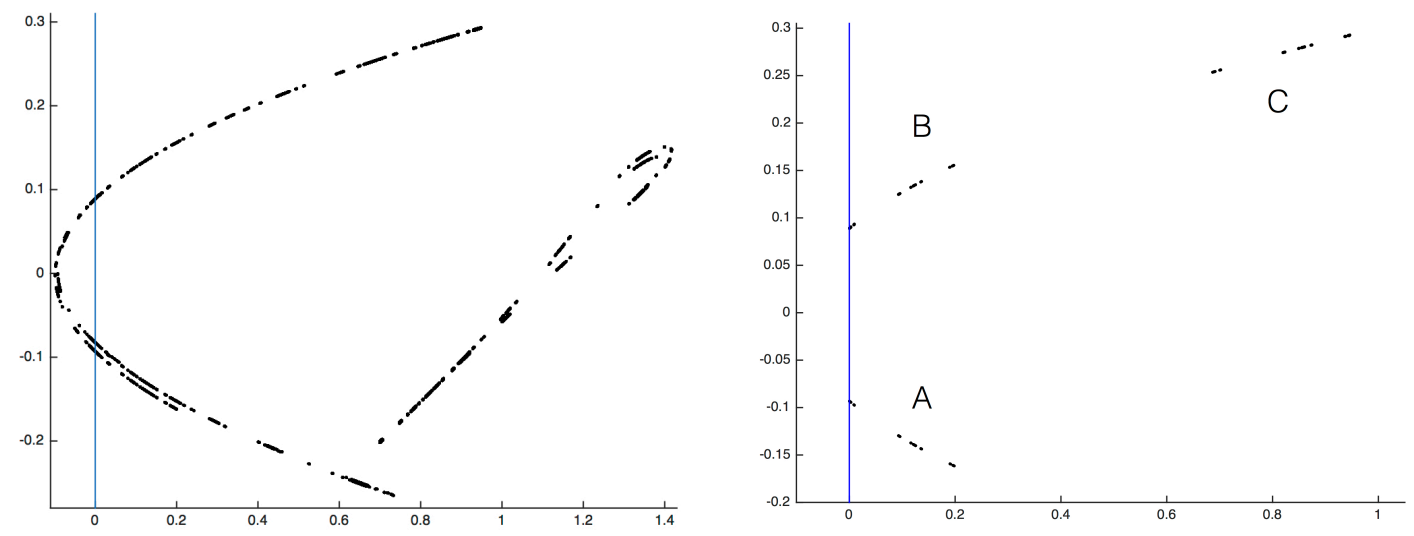

Figure 6: (a) A sample index pair for the full re-injected system. (b) Index pair for the cuspidal horseshoe without re-injection computed starting from the entire set of recurrent boxes.

symbols with transition matrix

$$
T=\left[\begin{array}{c|ccc} 
& A & B & C \\
\hline A & 0 & 1 & 1 \\
B & 0 & 1 & 1 \\
C & 1 & 0 & 0
\end{array}\right] .
$$

The entropy of this symbolic system is the natural logarithm of the spectral radius of $T$, which is approximately 0.48121182506 . It should be emphasized that simply verifying the transition graph on these regions is not enough to establish a rigorous lower bound on entropy; information extracted from computations on the Conley index of this index pair is required to show that the entropy of the transition graph is a lower bound on the entropy of the map; see [8].

All of the above computations are performed using three software packages. First, the construction of the outer approximation $\mathcal{F}$ on the grid $\mathcal{X}$, the processing of the graph of $\mathcal{F}$ for recurrent boxes and first return times, and the construction of index pairs are performed using Computational Dynamics Software (CDS) version 2.1 written by W. Kalies [19]. The computation of the homological Conley index maps on the index pairs is performed using Homcubes written by P. Pilarczyk [32]. Finally, the construction of a semiconjugate symbolic system used to calculate the rigorous lower bound on entropy is performed using Rigorous Analysis of Dynamical Systems (RADS) written by R. Frongillo [13].

Some comments on the computations are warranted. The number of subdivisions is chosen due to a limitation of the Homcubes software; 29 subdivisions is the most Homcubes allows. The computation of the map and the extraction of recurrent boxes are done concurrently and adaptively as described in [1] via algorithms that are linear time in the number of boxes and the size of the images. The first return time or minimal period of all recurrent boxes is computed with a quadratic time algorithm, and this calculation takes up much of the overall computational cost. This is why the computations are performed only up to $p=16$ in the full system. The computational expense of growing an isolating neighborhood depends not only on the number of boxes in the initial periodic set but also on the amount of hyperbolicity and other factors, and can be of moderate expense. Computing homology maps, symbolic dynamics, and entropy require relatively very little computation time.

\subsection{Final Remarks}

Theorem 1 states that for a certain open neighborhood of vector fields, the dynamics of the Poincaré map in a neighborhood of the origin is conjugate to that of the model of the re-injected cuspidal horseshoe in Section 1.1. In Equations (5) and (6) we derive an expansion of the Poincaré map. After rescaling and choosing some convenient values for the parameters as well as dropping higher-order terms, we obtain the system in Equation (71) on which we perform the computational analysis. As shown in Section 3, there is a narrow region of parameters in which Theorem 1 applies. We do not assert that the theorem applies for the choice of parameters in (71), nor do we include estimates of the higher order terms that occur in the Poincare map. Therefore, the computations we perform are rigorous only for the map (71) itself and not for any Poincaré map of a vector field with a re-injected cuspidal horseshoe. If an explicit vector field exhibiting 
a re-injected cuspidal horseshoe were known, one could potentially use rigorous numerical techniques, such as those in [42], to study the dynamics directly. However, it is not easy to obtain such an explicit vector field and is beyond the scope of this article.

However, as stated above, the computational results produce a rigorous semiconjugacy from the map (71) to a symbolic system using the Conley index as a validation tool. The symbolic system provides a lower bound on topological entropy of approximately 0.643. Since the multivalued map is an outer approximation, it is not enough to simply compute a transition graph on boxes. Even in this case of an explicit two-dimensional map, obtaining such a semiconjugacy is difficult without some form of rigorous computational analysis. Other rigorous computational methods could possibly be applied, c.f. [27] and [14], but such methods are typically more computationally intensive (as well as being more restrictive in the systems to which they can be applied), and in this setting the potentially finer information they provide is not necessary to demonstrate the effect of re-injection on the dynamics.

In the case of the restricted map $\varphi^{+}: S^{+} \rightarrow S^{+}$, we are able to compute a rigorous semiconjugacy onto a shift $\sigma_{T}$ on three symbols with transition matrix $T$ and obtain a lower bound on entropy of approximately 0.48121182506 . We conjecture that $\varphi^{+}$should actually be conjugate to the shift map $\sigma_{T}$, in which case this lower bound on entropy is also an upper bound. Figure 5(b) shows that the entropy of $\varphi^{+}$(lower magenta line) reaches this value when periodic boxes with minimal period at most 10 are used to build an isolating neighborhood and remains steady when higher periods up to 16 are included.

Figure 7 illustrates the symbolic dynamics. Let us assume that the choice of parameters in $\varphi^{ \pm}$are such that the map is conjugate to the geometric model of a re-injected cuspidal horseshoe, i.e. $\varphi^{ \pm}$is conjugate to a map $\Phi$ that satisfies properties (i)-(vii) in Section 1.1. This assumption essentially says that the map $\varphi^{+}$ has a thin enough image to act like a one-dimensional map. Under this assumption, Property (iv) implies that the map restricted to either of the "vertical" strips, the left strip containing regions $A$ and $B$ or the right one containing region $C$, is horizontally expanding on leaves of an invariant foliation. Moreover, as a consequence of the hyperbolicity property (vii), the map restricted to a neighborhood of the invariant set intersected with one of the regions $A, B$ or $C$ is vertically contracting on each leaf. This implies that the semiconjugacy onto the shift $\sigma_{T}$ given by the itinerary map is injective and hence is a conjugacy. Note that surjectivity could not be established without the computational Conley index results, and indeed there are parameter values for which $\varphi^{+}$does not have complicated dynamics.

These results, as shown in Figure 5, indicate that the dynamics with re-injection is indeed richer than the dynamics of $\varphi^{+}$alone. That is, for the map (71) the entropy of the full system is strictly larger than the entropy of the map $\varphi^{+}$without re-injection.

\section{References}

[1] Z. Arai, W.D. Kalies, H. Kokubu, K. Mischaikow, H. Oka, P. Pilarczyk, A database schema for the analysis of global dynamics of multiparameter systems, SIAM J. Appl. Dyn. Syst. 8, (2009), 757-789.

[2] H. Ban and W. Kalies, A computational approach to Conley's decomposition theorem, Journal of Computational and Nonlinear Dynamics 1 (2006), 312-319.

[3] P. Bonckaert, V. Naudot, Asymptotic properties of the Dulac map near a hyperbolic saddle in dimension three, Ann. Fac. Sci. Toulouse. Math. 6, (8), (2001), no. 4, 595-617.

[4] P. Bonckaert, V. Naudot, J. Yang, Linearization of hyperbolic resonant germs, Dynamical Systems: An international journal. 18, (1), March (2003), 69-88.

[5] S.N. Chow, B. Deng, B. Fiedler, Homoclinic bifurcation at resonant eigenvalues, Journ. Dynamics and Diff. Eq., 2, (1990), 177-244.

[6] B. Deng Homoclinic twisting bifurcation and cusp horseshoe maps, J. Dyn. Diff.Eq. 5, (1993), 417-467.

[7] S. Day, R. Frongillo, Sofic shifts via Conley index theory: computing lower bounds on recurrent dynamics for maps, Preprint, (2015).

[8] S. Day, R. Frongillo, R. Treviño, Algorithms for rigorous entropy bounds and symbolic dynamics, SIAM J. Appl. Dyn. Syst. 7, (2008), 1477-1506.

[9] S. Day, O. Junge, K. Mischaikow, Towards automated chaos verification, EQUADIFF $2003,157-162$. 


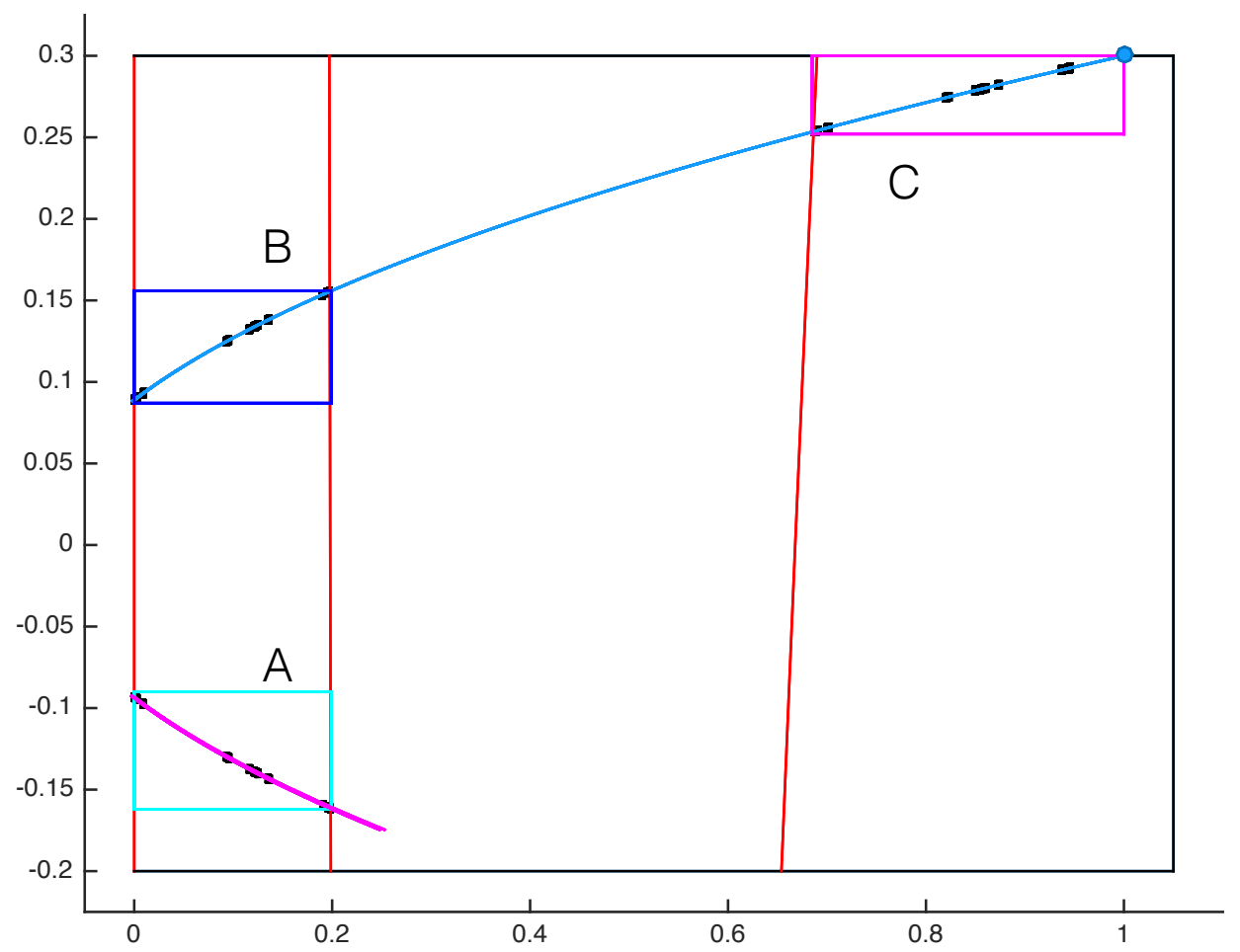

Figure 7: An illustration of the symbolic dynamics of $\varphi^{+}$. The region $C$ (magenta) maps across $A$ along the thin magenta strip. Regions $A$ (cyan) and $B$ (blue) map to the thin light blue strip which maps across both regions $B$ and $C$. The red curves are the line $\{x=0\}$ and its preimage under $\varphi^{+}$. The black boxes are the index pair in Figure 6. These boxes are enlarged to make them visible.

[10] M. Dellnitz, A. Hohmann, O. Junge, M. Rumpf, Exploring invariant sets and invariant measures, Chaos 7, (1997), 221-228.

[11] M. Fontaine, Nonlinear Phenomena from a Re-Injected Horseshoe, Ph.D. dissertation., Florida Atlantic University, (2016).

[12] J. Franks and D. Richeson, Shift equivalence and the Conley index, Trans. Amer. Math. Soc., 352 (2000), 3305-3322.

[13] R. Frongillo, Rigorous Analysis of Dynamical Systems (RADS), private communication, 2015.

[14] Z Galias and P Zgliczynski, Abundance of homoclinic and heteroclinic orbits and rigorous bounds for the topological entropy for the Hénon map, Nonlinearity, 14, (2001), p. 909.

[15] M. Hirsch, C. Pugh, M. Shub, Invariant Manifolds, Lect. Notes Math. 583 Springer 1977.

[16] A.J. Homburg, Global Aspects of Homoclinic Bifurcations of Vector Fields, Memoirs A.M.S. 578, (1996).

[17] A.J. Homburg, H. Kokubu, M. Krupa, The cusp horseshoe and its bifurcations in the unfolding of an inclination-flip homoclinic orbit, Ergod. Th. \& Dynam. Sys. 14 (1994), 667-693.

[18] T. Kaczynski, K. Mischaikow, and M. Mrozek, Computational Homology, Appl. Math. Sci. 157, SpringerVerlag, New York, 2004.

[19] W.D. Kalies, Computational Dynamics Software (CDS) ver. 2.1, available online at http://math.fau.edu/kalies/CDS, 2016.

[20] W.D. Kalies, K. Mischaikow, R.C.A.M. VanderVorst, An algorithmic approach to chain recurrence, Found. Comput. Math. 5, (2005), 409-449. 
[21] M. Kisaka, H. Kokubu, K. Oka, Bifurcations to N-homoclinic orbits and N-periodic orbits in vector fields, Journ. Dynamics and Diff. Eq. 5, (1993), 305-357.

[22] K. Mischaikow and M. Mrozek, Conley index, in Handbook of Dynamical Systems, Vol. 2, North-Holland, Amsterdam, 2002, 393-460.

[23] J. Moser. Stable and Random Motions in Dynamical Systems. Annals of Math. Studies. Princeton University Press, 1973.

[24] M. Martens, V. Naudot, and J. Yang, A strange attractor with large entropy in the unfodling of a low resonant degenerate homoclinic orbit. Intern. Journ. of Bifurcation $\&$ Chaos 16, No 12, (2006), 3509-3522.

[25] V. Naudot, Strange attractor in the unfolding of an inclination-flip homoclinic orbit, Ergod. Th. E Dynam. Syst. 16, (1996), 1071-1086.

[26] V. Naudot, A strange attractor in the unfolding of an orbit-flip homoclinic orbit, Dynamical Systems: An International Journal. 17, (1), (2002), 45-63.

[27] S. Newhouse, M. Berz, J. Grote, and K. Makino, On the Estimation of Topological Entropy on Surfaces Contemporary Mathematics, 469, (2008), 243-270.

[28] S. Nii, $N$-homoclinic orbits bifurcations for homoclinic orbits changing their twisting, J.Dynam. Diff. Eqs. 2, (2), (1996), 549-572.

[29] V. Naudot and J. Yang, Linearization of families of germs, Dyn. Syst. 23, (2008), no. 4, 467-489.

[30] J. Palis, F. Takens. Hyperbolicity and Sensitive Chaotic Dynamics at Homoclinic Bifurcations. Fractal Dimensions and infinitely many Attractors, Cambridge University Press 1993.

[31] J. Palis, W. de Melo, Geometric Theory of Dynamical Systems: An Introduction, Springer Verlag 1980.

[32] P. Pilarczyk, Homology Computation Software, Jagiellonian University, available online through the Computational Homology Project (Chomp) at http://chomp.rutgers.edu/software/, 1998.

[33] J. W. Robbin and D. Salamon, Dynamical systems, shape theory and the Conley index, Ergod. Th. \& Dynam. Syst., 8 (Charles Conley Memorial Issue), (1988), 375-393.

[34] R. Roussarie, C. Rousseau, Almost planar homoclinic loops in $\mathbb{R}^{3}$. J. Differential Equations 126, (1996), no. $1,147$.

[35] M.R. Rychlik, Lorenz attractors through Shil'nikov-type bifurcation. Part I, Ergod. Th. \& Dynam. Syst. 10, (1990), 793-821.

[36] B. Sandstede, Verzweigungstheorie homokliner Verdopplungen, Ph-d thesis, University of Stuttgart (1993).

[37] S. Smale, Differential dynamical systems, Bull. Am. Math. Soc.73, (1967), 747-817.

[38] V. Naudot, Bifurcations homoclines des champs de vecteurs en dimension trois, Thèse de l'Université de Bourgogne, Dijon (1996).

[39] L.P. Shil'nikov, A case of the existence of a countable number of periodic motions, Soviet Math. Dokl. 6, (1965), 163-166.

[40] A. Szymczak, The Conley index and symbolic dynamics, Topology 35 (1996), 287-299. [24]

[41] A. Szymczak, A combinatorial procedure for finding isolating neighborhoods and index pairs, Proc. Roy. Soc. Edinburgh Sect. A 127 (1997), pp. 1075-1088.

[42] W. Tucker, The Lorenz attractor exists, C. R. Acad. Sci. Paris Sér. I Math., 328, (1999), p. 1197-1202. 